\title{
BPS black holes, the Hesse potential, and the topological string
}

\author{
G.L. Cardoso, ${ }^{a}$ B. de $\mathbf{W i t}^{b, c}$ and S. Mahapatra ${ }^{d}$ \\ ${ }^{a}$ CAMGSD, Departamento de Matemática, Instituto Superior Técnico, \\ Av. Rovisco Pais, 1049-001 Lisboa, Portugal \\ ${ }^{b}$ Institute for Theoretical Physics, Utrecht University, \\ Leuvenlaan 4, 3584 CE Utrecht, The Netherlands \\ ${ }^{c}$ Nikhef Theory Group, \\ Science Park 105, 1098 XG Amsterdam, The Netherlands \\ ${ }^{d}$ Physics Department, Utkal University, \\ Bhubaneswar 751004 , India \\ E-mail: gcardoso@math.ist.utl.pt, B.deWit@uu.nl, swapna@iopb.res.in
}

Abstract: The Hesse potential is constructed for a class of four-dimensional $N=2$ supersymmetric effective actions with S- and T-duality by performing the relevant Legendre transform by iteration. It is a function of fields that transform under duality according to an arithmetic subgroup of the classical dualities reflecting the monodromies of the underlying string compactification. These transformations are not subject to corrections, unlike the transformations of the fields that appear in the effective action which are affected by the presence of higher-derivative couplings. The class of actions that are considered includes those of the FHSV and the STU model. We also consider heterotic $N=4$ supersymmetric compactifications. The Hesse potential, which is equal to the free energy function for BPS black holes, is manifestly duality invariant. Generically it can be expanded in terms of powers of the modulus that represents the inverse topological string coupling constant, $g_{\mathrm{s}}$, and its complex conjugate. The terms depending holomorphically on $g_{\mathrm{s}}$ are expected to correspond to the topological string partition function and this expectation is explicitly verified in two cases. Terms proportional to mixed powers of $g_{\mathrm{s}}$ and $\bar{g}_{\mathrm{s}}$ are in principle present.

Keywords: Supersymmetry and Duality, Black Holes in String Theory, Extended Supersymmetry, Topological Strings

ArXiv EPRINT: 1003.1970 


\section{Contents}

$\begin{array}{llr}1 & \text { Introduction } & 1\end{array}$

2 The Hesse potential 3

$\begin{array}{lll}3 & \text { S- and T-dualities } & 7\end{array}$

4 Performing the Legendre transform $\quad 11$

5 Heterotic $N=4$ supersymmetric string compactifications 16

6 The Hesse potential at second order $\quad 19$

7 The Hesse potential for specific models 22

\section{Introduction}

Higher-order curvature corrections to four-dimensional $N=2$ supersymmetric Wilsonian actions are known to affect the duality transformations of the moduli fields. The full transformation rules turn out to be much more complicated than their counterpart at the two-derivative level. An additional complication arises at the level of the associated 1PI effective action, where also non-holomorphic terms need to be incorporated in order to obtain physical results that reflect the duality invariance. These issues have been discussed in the context of four-dimensional $N=2$ BPS black holes [1,2], where it was described how to incorporate non-holomorphic corrections into the free energy of BPS black holes in the presence of a Weyl background. This free energy turns out to be given by the generalized Hesse potential. Unlike the effective action, the Hesse potential is defined in terms of variables whose duality transformations are not subject to the deformations induced by higher-derivative couplings. The relation between the Hesse potential and the effective action involves a Legendre transform. The Hesse potential can be regarded as the 'Hamiltonian' version of the effective Lagrangian, and is invariant under possible duality transformations. This is comparable to the generic situation for (abelian) gauge theories and electric/magnetic dualities, where the Lagrangian is in general not invariant under these dualities, while the Hamiltonian is invariant. Also the Lagrangian and the Hamiltonian are related by a Legendre transform and they are expressed in terms of different dynamical variables.

It is suggestive to assume that the Hesse potential is directly related to the partition function of the topological string [3]. The moduli of the topological string partition function correspond to moduli of the underlying Calabi-Yau moduli space and their duality 
transformations are defined in terms of monodromy transformations of the Calabi-Yau period vector. These transformations are thus directly related to the transformations found at the two-derivative level of the effective action. Therefore, both the topological string partition function and the Hesse potential are expressed in terms of variables that transform identically under the dualities, and moreover, both are duality invariant. In addition, it has been established that certain string amplitudes are related to the twisted partition functions of the topological string $[3,4]$. String amplitudes correspond to connected field theory graphs, and therefore these amplitudes must be encoded in both the corresponding effective action and in the Hesse potential. Consequently the topological string is contained in the Hesse potential. This leaves the possibility that the Hesse potential contains more information than just the topological string in view of the fact that its dependence on the topological string coupling, $g_{\mathrm{s}}$, which is inversely proportional to one of the complex moduli, is in principle not holomorphic.

In general it is not possible to carry out the Legendre transform explicitly at the level of a full effective action. This paper is therefore devoted to carrying out the Legendre transform by iteration in order to subsequently study the possible relation between the Hesse potential and the partition function of the topological string. Hence the BPS black holes will only play an ancillary role in this work. The Legendre transform leads to new variables for the Hesse potential which, under duality, transform precisely like the fields used in the topological string. Unfortunately only in a few cases exact expressions are known for the topological string, and for the effective action there is even less data. Consequently we will have to rely on a restricted number of models with a high degree of symmetry. However, in principle, our results will also be relevant for models without duality symmetries.

For a specific class of $N=2$ models, which includes the FHSV [5] and the STU model $[6,7]$, we explicitly compute the Hesse potential in terms of these new variables, up to second order in the Weyl background. In the context of the FHSV model, we show that the Legendre transform reproduces the associated non-holomorphic genus-2 partition function of the topological string [8], starting from the expressions found in [2]. In addition, we consider $N=4$ supersymmetric models in this $N=2$ description, for which we obtain more detailed information on the higher-order contributions to the Hesse potential.

Some time ago it has been argued [1] that the exponent of the Hesse potential appears in a fully duality invariant extension of the OSV integral [9]. A semiclassical evaluation of this extension reproduces the original OSV integral with an additional measure factor. At the semiclassical level this modified integral correctly reproduces all known results for (large) black holes from both macroscopic and microscopic perspectives. Beyond the semiclassical approximation the role of this integral has not been fully established as yet.

The results of this paper clarify a number of issues in the relation between the effective action and the Hesse potential. The latter depends on a modulus that corresponds to the inverse topological string coupling constant $g_{\mathrm{s}}$ and on its complex conjugate. As it turns out, the topological string partition functions are recovered when restricting to those terms in the Hesse potential that depend holomorphically on $g_{\mathrm{s}}$, at least for genus $g \leq 2$. This sector of the Hesse potential is separately consistent with respect to duality. In view of the earlier discussion this result is not unexpected, but the present lack of data on the 
higher-derivative terms in the effective actions forms an obstacle for uncovering the more conceptual aspects of the relation between the Hesse potential and the topological string partition function. In principle, the Hesse potential will also contain terms proportional to mixed powers of $g_{\mathrm{s}}$ and $\bar{g}_{\mathrm{s}}$. It is important to realize that these are not primarily induced by the non-holomorphic corrections associated with non-local terms in the effective action, but they are present as a result of the Legendre transform. We observe that, at genus 2, these mixed terms can be absorbed by suitable contributions from the effective action. Without detailed knowledge of the latter, it is not clear how to establish this in some generality, especially because the consequences of (partially) absorbing these terms can only be seen at higher genus. Perhaps the analysis can be strengthened eventually by taking into account information on the asymptotic behaviour of the functions involved. Admittedly, the situation remains rather complex, but it is clear that considerable progress can be made on the basis of the case studies considered in this paper.

This paper is organized as follows. In section 2, after reviewing the construction of the Hesse potential in the presence of non-holomorphic terms, we introduce new variables $\tilde{Y}^{I}$ for the Hesse potential that transform under duality according to the classical transformation rules. Section 3 contains a brief review of the consequences of S- and T-duality invariance for a class of $N=2$ models that contain the FHSV and the STU model. In section 4 we construct the variables $\tilde{Y}^{I}$ for this class of $N=2$ models. We derive a set of equations that these new variables have to satisfy, and we solve them iteratively in the Weyl background, up to second order. We verify that these new parameters satisfy the required duality properties. Section 5 deals with models corresponding to heterotic $N=4$ compactifications, for which we derive all-order results. In section 6 we compute the Hesse potential for the more generic case, expressed in the new variables, to second order. Section 7 summarizes the situation for specific models and compares the results to the twisted partition functions of the topological string. In two cases we demonstrate that these partition functions can be reproduced by the corresponding terms in the Hesse potential that depend holomorphically on $g_{\mathrm{s}}$.

\section{The Hesse potential}

For $N=2$ supergravity the part of the Lagrangian pertaining to the vector supermultiplets is encoded in a holomorphic function $F$ of the complex scalar fields $X^{I}$ belonging to these multiplets, which is homogeneous of second degree. Here the index $I=0,1, \ldots, n$ labels the various vector multiplets. The vector multiplets have an optional coupling to the square of the Weyl tensor, which can be encoded in the function $F$ by introducing a dependence on another complex scalar field equal to the square of the anti-selfdual antisymmetric auxiliary field that constitutes the lowest-weight field of the so-called Weyl supermultiplet. All these scalars are defined projectively, but in the context of BPS black holes suitably normalized fields have been introduced denoted by $Y^{I}$ and $\Upsilon$ [10]. In terms of these fields the attractor equations for BPS black holes take the form,

$$
Y^{I}-\bar{Y}^{I}=\mathrm{i} p^{I}, \quad F_{I}-\bar{F}_{I}=\mathrm{i} q_{I}, \quad \Upsilon=-64,
$$


where $F_{I}$ denotes the derivative of the function $F$ with respect to $Y^{I}$ and $\bar{F}_{I}$ is its complex conjugate. ${ }^{1}$ The first two atractor equations can be obtained from extremizing the BPS free energy. This observation will be relevant in the sequel.

The above does not yet account for the presence of non-holomorphic modifications. These modifications signal departures from the Wilsonian action that originate from integrating out the massless modes in order to obtain the full effective action. This integration gives rise to non-local terms in the corresponding supergravity action. Unfortunately not much is known about these non-localities, except that they are often required to preserve physical symmetries that cannot be fully realized at the level of the Wilsonian action. An early example of this phenomenon can be found in [11], where it was demonstrated that the gauge coupling constants in heterotic string compactifications are moduli dependent with non-holomorphic corrections. Also in the context of BPS black holes the need for non-holomorphic modifications has been demonstrated to ensure that the 'period vector' $\left(Y^{I}, F_{I}\right)$ transforms consistently under S-duality [12]. When these modifications are taken into account an S-duality invariant entropy is obtained. The results of this analysis are in accord with the results for the non-holomorphic terms found in the corresponding effective action [13]. More recently, it has been shown [14, 15] how the same results emerge from a semiclassical approximation of the microscopic degeneracy formula for $N=4$ dyons [15-21].

In order to ensure that the attractor equations will still follow from a variational principle in the presence of non-holomorphic corrections, it turns out that these corrections must be encoded in a real and homogeneous function of second degree denoted by $\Omega(Y, \bar{Y}, \Upsilon, \bar{\Upsilon})$, which is incorporated into the function $F$ in the following way $[2,14]$,

$$
F=F^{(0)}(Y, \Upsilon)+2 \mathrm{i} \Omega(Y, \bar{Y}, \Upsilon, \bar{\Upsilon})
$$

The attractor equations (2.1) retain the same form, irrespective of the presence of these non-holomorphic terms. Although the explicit couplings in the Lagrangian corresponding to this modification are unknown, it turned out that important progress can be made without first constructing the full effective action. When the function $\Omega$ is harmonic, i.e., when it can be written as the sum of a holomorphic and an anti-holomorphic function, then one may simply absorb the holomorphic part into the first term. The anti-holomorphic part will then not contribute as it will vanish under the holomorphic derivatives which enter the attractor equations as well as the black hole entropy. Consequently we can incorporate the $\Upsilon$-dependent terms in $F^{(0)}$ into $\Omega$. In that case $F^{(0)}(Y)$ will no longer depend on $\Upsilon$, and will refer to the classical contribution that pertains to the part of the Lagrangian quadratic in space-time derivatives.

In this context there exists the notion of a BPS free energy, which is defined as follows [1],

$$
\mathcal{F}(Y, \bar{Y}, \Upsilon, \bar{\Upsilon})=-\mathrm{i}\left(\bar{Y}^{I} F_{I}-Y^{I} \bar{F}_{I}\right)-2 \mathrm{i}\left(\Upsilon F_{\Upsilon}-\bar{\Upsilon} \bar{F}_{\bar{\Upsilon}}\right)
$$

where $F_{\Upsilon}=\partial F / \partial \Upsilon$. We recall that each of the two terms in (2.3) transform as a function under electric/magnetic duality. This free energy, whose existence seems desirable based

\footnotetext{
${ }^{1}$ Hence $\bar{F}_{I}$ equals the derivative of $\bar{F}$ with respect to $\bar{Y}^{I}$. We refrain from distinguishing holomorphic and anti-holomorphic derivatives, $\partial / \partial Y^{I}$ and $\partial / \partial \bar{Y}^{I}$, by the use of different types of indices.
} 
on semiclassical arguments, enters the BPS entropy function $\Sigma$, defined by

$$
\Sigma(Y, \bar{Y}, p, q)=\mathcal{F}(Y, \bar{Y})-q_{I}\left(Y^{I}+\bar{Y}^{I}\right)+p^{I}\left(F_{I}+\bar{F}_{I}\right),
$$

where the black hole charges $q_{I}$ and $p^{I}$ couple to the corresponding electro- and magnetostatic potentials at the horizon, which are equal to [22],

$$
\phi^{I}=Y^{I}+\bar{Y}^{I}, \quad \chi_{I}=F_{I}+\bar{F}_{I} .
$$

In [2] we have indicated how these expressions are consistent with electric/magnetic duality. Requiring stationarity of the entropy function $\Sigma$ with respect to $Y^{I}$ leads directly to the attractor equations (2.1) while the value of $\Sigma$ at the attractor point defines the macroscopic (field-theoretic) entropy divided by $\pi$. In the absence of non-holomorphic modifications, this entropy has been shown [10] to coincide with Wald's entropy based on a Noether charge [23-25]. Under electric/magnetic duality the charges $\left(p^{I}, q_{I}\right)$ and the 'period vector' $\left(Y^{I}, F_{I}\right)$ transform under symplectic (real) rotations, and so do the potentials $\left(\phi^{I}, \chi_{I}\right)$.

In the presence of higher-order derivative actions in the effective action, the original complex fields $Y^{I}$ transform in a complicated way under electric/magnetic duality. Therefore it is advantageous to consider a variable change to the real coordinates $\phi^{I}$ and $\chi_{I}$, which transform linearly under dualities. This conversion is well defined whenever $\operatorname{det}\left[F_{I J}-\bar{F}_{I J}\right] \neq 0$, where $F_{I J}$ denotes the second derivative of $F$ with respect to $Y^{I}$ and $Y^{J}$. As we shall demonstrate shortly, the so-called Hesse potential, defined as the Legendre transform of a linear combination of the imaginary part of $F$ and $\Omega$ with respect to the imaginary part of the $Y^{I}$, is a function of $\phi^{I}$ and $\chi_{I}$. It is a generalization of the Hesse potential defined in the context of special geometry [26, 27]. To perform the conversion to real variables $\phi^{I}$ and $\chi_{I}$, we first decompose $Y^{I}$ and $F_{I}$ into their real and imaginary parts,

$$
Y^{I}=\frac{1}{2}\left(\phi^{I}+\mathrm{i} u^{I}\right), \quad F_{I}=\frac{1}{2}\left(\chi_{I}+\mathrm{i} v_{I}\right) .
$$

The real parametrization is obtained by taking $\left(\phi^{I}, \chi_{I}, \Upsilon, \bar{\Upsilon}\right)$ instead of $\left(Y^{I}, \bar{Y}^{I}, \Upsilon, \bar{\Upsilon}\right)$ as the independent variables. Although $\Upsilon$ is a spectator, note that the inversion of $\chi_{I}=$ $\chi_{I}(\phi, u, \Upsilon, \bar{\Upsilon})$ gives $\operatorname{Im} Y^{I}=u^{I}(\phi, \chi, \Upsilon, \bar{\Upsilon})$. To compare partial derivatives in the two parametrizations, we need,

$$
\begin{aligned}
\left.\frac{\partial}{\partial \phi^{I}}\right|_{u} & =\left.\frac{\partial}{\partial \phi^{I}}\right|_{\chi}+\left.\frac{\partial \chi_{J}(\phi, u, \Upsilon, \bar{\Upsilon})}{\partial \phi^{I}} \frac{\partial}{\partial \chi_{J}}\right|_{\phi}, \\
\left.\frac{\partial}{\partial u^{I}}\right|_{\phi} & =\left.\frac{\partial \chi_{J}(\phi, u, \Upsilon, \bar{\Upsilon})}{\partial u^{I}} \frac{\partial}{\partial \chi_{J}}\right|_{\phi}, \\
\left.\frac{\partial}{\partial \Upsilon}\right|_{\phi, u} & =\left.\frac{\partial}{\partial \Upsilon}\right|_{\phi, \chi}+\left.\frac{\partial \chi_{I}(\phi, u, \Upsilon, \bar{\Upsilon})}{\partial \Upsilon} \frac{\partial}{\partial \chi_{I}}\right|_{\phi} .
\end{aligned}
$$

The homogeneity is preserved under the reparametrization because $\chi(\phi, u, \Upsilon, \bar{\Upsilon})$ is a homogeneous function of first degree. This results in the equality,

$$
\begin{aligned}
& \left.\phi^{I} \frac{\partial}{\partial \phi^{I}}\right|_{u}+\left.u^{I} \frac{\partial}{\partial u^{I}}\right|_{\phi}+\left.2 \Upsilon \frac{\partial}{\partial \Upsilon}\right|_{\phi, u}+\left.2 \bar{\Upsilon} \frac{\partial}{\partial \bar{\Upsilon}}\right|_{\phi, u} \\
& \quad=\left.\phi^{I} \frac{\partial}{\partial \phi^{I}}\right|_{\chi}+\left.\chi_{I} \frac{\partial}{\partial \chi_{I}}\right|_{\phi}+\left.2 \Upsilon \frac{\partial}{\partial \Upsilon}\right|_{\phi, \chi}+\left.2 \bar{\Upsilon} \frac{\partial}{\partial \bar{\Upsilon}}\right|_{\phi, \chi} .
\end{aligned}
$$


The Hesse potential is defined as the Legendre transform of $4(\operatorname{Im} F-\Omega)$ with respect to $u^{I}=2 \operatorname{Im} Y^{I},^{2}$

$$
\mathcal{H}(\phi, \chi, \Upsilon, \bar{\Upsilon})=4 \operatorname{Im} F(Y, \bar{Y}, \Upsilon, \bar{\Upsilon})-4 \Omega(Y, \bar{Y}, \Upsilon, \bar{\Upsilon})-\chi_{I} u^{I}
$$

which is a homogeneous function of second degree. Note that $\delta \mathcal{H}=v_{I} \delta \phi^{I}-u^{I} \delta \chi_{I}$, which shows that the attractor equations (2.1) take the form,

$$
\frac{\partial \mathcal{H}}{\partial \phi^{I}}=q_{I}, \quad \frac{\partial \mathcal{H}}{\partial \chi_{I}}=-p^{I}
$$

These equations follow from requiring that the entropy function

$$
\Sigma(\phi, \chi, p, q)=\mathcal{H}(\phi, \chi, \Upsilon, \bar{\Upsilon})-q_{I} \phi^{I}+p^{I} \chi_{I},
$$

is stationary. Comparing this result to the entropy function (2.4) indicates that the Hesse potential is just the BPS free energy (2.3). Indeed, using the homogeneity properties of $F$ and $\Omega$, we establish the relation,

$$
\mathcal{H}(\phi, \chi, \Upsilon, \bar{\Upsilon})=-\mathrm{i}\left(\bar{Y}^{I} F_{I}-Y^{I} \bar{F}_{I}\right)-2 \mathrm{i}\left(\Upsilon F_{\Upsilon}-\bar{\Upsilon} \bar{F}_{\bar{\Upsilon}}\right)=\mathcal{F}(Y, \bar{Y}, \Upsilon, \bar{\Upsilon})
$$

Substituting the result of the attractor equations into the entropy function thus yields the macroscopic BPS entropy, just as before (irrespective of the non-holomorphic modification). In the spirit of [9] it has been proposed that the integral over $\exp [\Sigma(\phi, \chi, p, q)]$ yields the entropy for BPS black holes, and this proposal has been verified in a variety of cases at the semiclassical level [1]. Some of its implications have also successfully been confronted with microscopic counting data, mainly from heterotic $N=4$ supersymmetric models [1417, 28]. ${ }^{3}$ However, we should note that no exact results are available as yet.

Under duality invariances the complex variables $Y^{I}$ transform in a complicated way, which can be studied order-by-order in $\Upsilon[2]$. To explicitly establish the invariance of the BPS free energy is thus cumbersome, as both the transformation rules and the expression for the free energy take the form of a power series in $\Upsilon$. This was analyzed extensively in [2], where arguments were put forward that show that the duality invariance persists in the presence of the non-holomorphic modifications. The Hesse potential depends on fields $\left(\phi^{I}, \chi_{I}\right)$ that transform under the dualities with a real symplectic rotation, just as the charges $\left(p^{I}, q_{I}\right)$. These rotations, referred to as monodromies, are fixed from the start and cannot be subject to any iterative procedure (because of the integer-valued charge lattice). Therefore, it is in principle easier to consider the duality invariance of the Hesse potential, but this quantity has to be evaluated by a Legendre transform which cannot be explicitly performed and requires an iterative procedure.

In this paper we will evaluate the first few terms of the expansion of the Hesse potential in terms of $\Upsilon$ for a class of $N=2$ effective actions with S- and T-duality. Subsequently, we will verify the duality invariance of the terms in the expansion and compare them to results known for the topological string partition function. It is rather convenient to do this in

\footnotetext{
${ }^{2}$ See [1]; note that the conventions of this paper are not the same.

${ }^{3}$ For an $N=2$ application, see [29].
} 
terms of different variables than the real potentials $\left(\phi^{I}, \chi_{I}\right)$. Namely, we will re-express the $\left(\phi^{I}, \chi_{I}\right)$, which incorporate all the terms of the action, in terms of new complex fields denoted by $\tilde{Y}^{I}$, which will coincide precisely with the fields $Y^{I}$ that one would obtain from $\left(\phi^{I}, \chi_{I}\right)$ by using only the lowest-order holomorphic function $F^{(0)}$. Hence the identification proceeds as follows,

$$
\begin{aligned}
2 \operatorname{Re} Y^{I} & =\phi^{I}=2 \operatorname{Re} \tilde{Y}^{I} \\
2 \operatorname{Re} F_{I}(Y, \bar{Y}, \Upsilon, \bar{\Upsilon}) & =\chi_{I}=2 \operatorname{Re} F_{I}^{(0)}(\tilde{Y}) .
\end{aligned}
$$

At the classical level $\tilde{Y}^{I}=Y^{I}$, but in higher orders the relation between these moduli is complicated and will depend on $\Upsilon$. The crucial point is that the duality transformations for the fields $\tilde{Y}^{I}$ will be independent of $\Upsilon$ and its complex conjugate, unlike the transformations of the fields $Y^{I}$, which depend non-trivially on $\Upsilon, \bar{\Upsilon}$. Therefore, the moduli $\tilde{Y}^{I}$ are expected to be the appropriate variables for the topological string. We note that the passage from the supergravity (or effective action) variables $Y^{I}$ to the topological string variables $\tilde{Y}^{I}$ induces a change of complex structure (which is thus not primarily induced by the nonholomorphic terms contained in the function $\Omega$ ). This will become evident in section 4 , where we compute the change for a class of models with a high degree of symmetry. Observe that the left-hand side of the second equation (2.13) depends explicitly on the function $\Omega$, whereas the relation between $(\phi, \chi)$ and the $\tilde{Y}^{I}$ represents a simple change of variables. As it turns out, this change of variables facilitates the calculations that we will perform in later sections.

It is easy to verify that the covariant moduli proposed for the STU model in [30] do not fall in the same class as the moduli $\tilde{Y}^{I}$, simply because they do not satisfy (2.13). It remains to be seen what the relation between the two sets of covariant variables implies. At any rate, the variables used in this paper exist generally, outside the context of a specific model.

\section{S- and T-dualities}

Following [2] we consider a class of models for which the lowest-order contribution of the action is encoded in the holomorphic, homogeneous function,

$$
F^{(0)}(Y)=-\frac{Y^{1} Y^{a} \eta_{a b} Y^{b}}{Y^{0}}
$$

where $a, b=2, \ldots, n$, and the symmetric matrix $\eta_{a b}$ is an $\mathrm{SO}(n-2,1)$ invariant metric of indefinite signature. To this expression we will add the homogeneous real function $\Omega$ as specified in (2.2), which, as explained in the previous section, encodes certain higher-order derivative as well as non-local interactions. Models of this type arise in type-II compactifications on Calabi-Yau three-folds that are K3 fibrations. The number $n$ depends on the particular model that one is considering. Both the FHSV [5] and the STU model $[6,7]$ belong to this class and have $n=11$ and $n=3$, respectively. For these models explicit information is available for the terms of higher-order in $\Upsilon[2,8]$. Furthermore we use the $N=2$ supergravity description to also consider a number of heterotic string compactifications with $N=4$ supersymmetry. 
In the absence of non-holomorphic corrections the function $F(Y, \Upsilon)$ takes the form of a loop expansion with $Y^{0}$ as a loop-counting parameter,

$$
F(Y, \Upsilon)=\mathrm{i}\left(Y^{0}\right)^{2} S T^{a} \eta_{a b} T^{b}+\Upsilon F^{(1)}(S, T)+\sum_{g=2}^{\infty} \frac{\Upsilon^{g}}{\left(Y^{0}\right)^{2 g-2}} F^{(g)}(S, T),
$$

with 'special coordinates' defined in the usual fashion,

$$
S=-\mathrm{i} Y^{1} / Y^{0}, \quad T^{a}=-\mathrm{i} Y^{a} / Y^{0} .
$$

In the context of type-II models based on K3-fibered Calabi-Yau three-folds, these special coordinates can be used to parametrize (half of) the moduli space of the associated string compactification. An expansion such as (3.2) is also relevant for the topological string on the same Calabi-Yau three-fold, where $Y^{0}$ is regarded as the inverse topological string coupling constant and the functions $F^{(g)}(S, T)$ are the genus- $g$ twisted partition functions. ${ }^{4}$ The latter acquire non-holomorphic corrections encoded in the holomorphic anomaly equation, whose structure is such that the holomorphic dependence on the topological string coupling constant is preserved [3]. As we already mentioned, non-holomorphic corrections are also required to realize the relevant symmetries of the effective action [11], and it seems likely that these two phenomena are in fact related. To clarify this in some detail was in fact one of the motivations for the work described here. However, we should stress that in spite of the fact that the same expansion (3.2) is relevant for both the effective action and for the topological string, the two expansions should not be identified, as was demonstrated in [2]. In the next section we will work out the precise correspondence in more detail by explicitly performing the Legendre transform for the Hesse potential, up to $g=2$.

In this section we will be reviewing the S- and T-duality transformations based on [2], where the effect of the dualities was determined for the partial derivatives of $\Omega$. For the models based on (3.1) the duality group is given by $\mathrm{SL}(2 ; \mathbb{Z}) \times \mathrm{O}(n-1,2 ; \mathbb{Z})$, where the first factor refers to the S-duality group and the second one to the T-duality group. When including the $\Upsilon$-dependent terms according to (2.2), only a subgroup may be realized. In [2] the requirements for the function $\Omega$ were derived based on the assumption that the invariance was realized for a suitable arithmetic subgroup. Under this group the $\left(Y^{I}, F_{I}\right)$ transform as follows under S-duality,

$$
\begin{array}{ll}
Y^{0} \rightarrow d Y^{0}+c Y^{1}, & F_{0} \rightarrow a F_{0}-b F_{1}, \\
Y^{1} \rightarrow a Y^{1}+b Y^{0}, & F_{1} \rightarrow d F_{1}-c F_{0}, \\
Y^{a} \rightarrow d Y^{a}-\frac{1}{2} c \eta^{a b} F_{b}, & F_{a} \rightarrow a F_{a}-2 b \eta_{a b} Y^{b},
\end{array}
$$

where $a, b, c, d$ are integer-valued parameters that satisfy $a d-b c=1$ which parametrize (a subgroup of $) \mathrm{SL}(2 ; \mathbb{Z})$.

For the T-duality group, general transformations are conveniently generated by products of a number of specific finite transformations. Those belonging to the $\mathrm{O}(n-2,1 ; \mathbb{Z})$

\footnotetext{
${ }^{4}$ Hence $F^{(g)}(Y)=\left(Y^{0}\right)^{2-2 g} F^{(g)}(S, T)$; when referring to the genus- $g$ partition functions in the text, we usually do not make a distinction between $F^{(g)}(Y)$ and $F^{(g)}(S, T)$.
} 
subgroup are manifest in the above description and do not need to be considered. Then there are $n-1$ abelian transformations generated by

$$
\begin{array}{lll}
Y^{0} \rightarrow Y^{0}, & F_{0} \rightarrow F_{0}+\lambda^{a} F_{a}+\lambda^{a} \eta_{a b} \lambda^{b} Y^{1}, \\
Y^{1} \rightarrow Y^{1}, & F_{1} \rightarrow F_{1}+2 \lambda^{a} \eta_{a b} Y^{b}-\lambda^{a} \eta_{a b} \lambda^{b} Y^{0}, \\
Y^{a} \rightarrow Y^{a}-\lambda^{a} Y^{0}, & F_{a} \rightarrow F_{a}+2 \eta_{a b} \lambda^{b} Y^{1},
\end{array}
$$

where the $\lambda^{a}$ are integers. The full $\mathrm{O}(n-1,2 ; \mathbb{Z})$ group is generated upon including also the following transformation,

$$
\begin{array}{ll}
Y^{0} \rightarrow F_{1}, & F_{0} \rightarrow-Y^{1} \\
Y^{1} \rightarrow-F_{0}, & F_{1} \rightarrow Y^{0}, \\
Y^{a} \rightarrow Y^{a}, & F_{a} \rightarrow F_{a},
\end{array}
$$

which squares to the identity.

In the case that $\Omega$ is suppressed in (2.2), it is straightforward to evaluate the behaviour of these transformations on the special coordinates $S$ and $T^{a}$, and on the remaining field $Y^{0}$. Under S-duality we find,

$$
S \rightarrow \frac{a S-\mathrm{i} b}{d+\mathrm{i} c S}, \quad T^{a} \rightarrow T^{a} \quad Y^{0} \rightarrow(d+\mathrm{i} c S) Y^{0} .
$$

The T-duality transformations (3.5) and (3.6) lead to, respectively,

$$
S \rightarrow S, \quad T^{a} \rightarrow T^{a}+\mathrm{i} \lambda^{a}, \quad T^{a} \rightarrow \frac{T^{a}}{T^{b} \eta_{b c} T^{c}} \quad Y^{0} \rightarrow T^{b} \eta_{b c} T^{c} Y^{0} .
$$

These S- and T-duality transformations become much more complicated when $\Omega$ is taken into account in (2.2). Insisting on the same symmetry (i.e., characterized by the same transformations acting on $\left(Y^{I}, F_{I}\right)$ ), or a subgroup thereof, severely restricts the $\Upsilon$-dependent contributions contained in $\Omega$. These restrictions take the form of prescribed transformation rules for the first-order derivatives $\Omega$ with respect to the fields. The crucial observation is, however, that the fields $\tilde{S}, \tilde{T}^{a}$ and $\tilde{Y}^{0}$, based on (2.13), with

$$
\tilde{S}=-\mathrm{i} \tilde{Y}^{1} / \tilde{Y}^{0}, \quad \tilde{T}^{a}=-\mathrm{i} \tilde{Y}^{a} / \tilde{Y}^{0},
$$

will still transform exactly as in (3.7) and (3.8). Of course, this is true provided the $\Upsilon$ dependent terms satisfy the correct symmetry properties. These will be summarized below.

Since the S- and T-duality transformations involve the derivatives $F_{I}$, we note the expressions,

$$
\begin{aligned}
& F_{0}=\frac{Y^{1}}{\left(Y^{0}\right)^{2}} Y^{a} \eta_{a b} Y^{b}-\frac{2 \mathrm{i}}{Y^{0}}\left[-Y^{0} \frac{\partial}{\partial Y^{0}}+S \frac{\partial}{\partial S}+T^{a} \frac{\partial}{\partial T^{a}}\right] \Omega, \\
& F_{1}=-\frac{1}{Y^{0}} Y^{a} \eta_{a b} Y^{b}+\frac{2}{Y^{0}} \frac{\partial \Omega}{\partial S}, \\
& F_{a}=-2 \frac{Y^{1}}{Y^{0}} \eta_{a b} Y^{b}+\frac{2}{Y^{0}} \frac{\partial \Omega}{\partial T^{a}},
\end{aligned}
$$

where we regard $\Omega$ as a function of $Y^{0}, S$ and $T^{a}$ (and their complex conjugates). 
With these results the S-duality transformations (3.4) take the form,

$$
\begin{aligned}
Y^{0} & \rightarrow \Delta_{\mathrm{S}} Y^{0} \\
Y^{1} & \rightarrow a Y^{1}+b Y^{0}, \\
Y^{a} & \rightarrow \Delta_{\mathrm{S}} Y^{a}-\frac{c}{Y^{0}} \eta^{a b} \frac{\partial \Omega}{\partial T^{b}},
\end{aligned}
$$

with

$$
\Delta_{\mathrm{S}}=d+\mathrm{i} c S
$$

On the special coordinates $S$ and $T^{a}$ these transformations extend the previous result (3.7),

$$
S \rightarrow \frac{a S-\mathrm{i} b}{\mathrm{i} c S+d}, \quad T^{a} \rightarrow T^{a}+\frac{\mathrm{i} c}{\Delta_{\mathrm{S}}\left(Y^{0}\right)^{2}} \eta^{a b} \frac{\partial \Omega}{\partial T^{b}}
$$

and we note the useful relations

$$
\frac{\partial S^{\prime}}{\partial S}=\Delta_{\mathrm{S}}{ }^{-2}, \quad \frac{1}{S+\bar{S}} \rightarrow \frac{\left|\Delta_{\mathrm{S}}\right|^{2}}{S+\bar{S}}=\frac{\Delta_{\mathrm{S}}^{2}}{S+\bar{S}}-\mathrm{i} c \Delta_{\mathrm{S}}
$$

Assuming that the above transformations constitute an invariance of the model, we require that the S-duality transformations of the $Y^{I}$ induce the expected transformations of the $F_{I}$ upon substitution. This leads to the following result, ${ }^{5}$

$$
\begin{aligned}
\left(\frac{\partial \Omega}{\partial T^{a}}\right)_{\mathrm{S}}^{\prime} & =\frac{\partial \Omega}{\partial T^{a}} \\
\left(\frac{\partial \Omega}{\partial S}\right)_{\mathrm{S}}^{\prime}-\Delta_{\mathrm{S}}^{2} \frac{\partial \Omega}{\partial S} & =\frac{\partial\left(\Delta_{\mathrm{S}}^{2}\right)}{\partial S}\left[-\frac{1}{2} Y^{0} \frac{\partial \Omega}{\partial Y^{0}}-\frac{\mathrm{i} c}{4 \Delta_{\mathrm{S}}\left(Y^{0}\right)^{2}} \frac{\partial \Omega}{\partial T^{a}} \eta^{a b} \frac{\partial \Omega}{\partial T^{b}}\right] \\
\left(Y^{0} \frac{\partial \Omega}{\partial Y^{0}}\right)_{\mathrm{S}}^{\prime} & =Y^{0} \frac{\partial \Omega}{\partial Y^{0}}+\frac{\mathrm{i} c}{\Delta_{\mathrm{S}}\left(Y^{0}\right)^{2}} \frac{\partial \Omega}{\partial T^{a}} \eta^{a b} \frac{\partial \Omega}{\partial T^{b}}
\end{aligned}
$$

The same reasoning applies to T-duality. Under the transformation (3.5) it follows from (3.10) that all the derivatives $\partial \Omega / \partial Y^{0}, \partial \Omega / \partial S$ and $\partial \Omega / \partial T^{a}$ must be invariant under integer shifts $T^{a} \rightarrow T^{a}+\mathrm{i} \lambda^{a}$. For the T-duality transformation (3.6) the analysis is more subtle. Using (3.10) we derive,

$$
\begin{aligned}
Y^{0} & \rightarrow \Delta_{\mathrm{T}} Y^{0}, \\
Y^{1} & \rightarrow \Delta_{\mathrm{T}} Y^{1}+\frac{2 \mathrm{i}}{Y^{0}}\left[-Y^{0} \frac{\partial \Omega}{\partial Y^{0}}+T^{a} \frac{\partial \Omega}{\partial T^{a}}\right], \\
Y^{a} & \rightarrow Y^{a}
\end{aligned}
$$

with

$$
\Delta_{\mathrm{T}}=T^{a} \eta_{a b} T^{b}+\frac{2}{\left(Y^{0}\right)^{2}} \frac{\partial \Omega}{\partial S}
$$

\footnotetext{
${ }^{5}(O)_{\mathrm{S}, \mathrm{T}}^{\prime}$ denotes the change of $O$ under S- or T-duality induced by the transformation of all the arguments on which $O$ depends.
} 
On the special coordinates the transformation (3.16) extends the previous result (3.8),

$$
\begin{aligned}
S & \rightarrow S+\frac{2}{\Delta_{\mathrm{T}}\left(Y^{0}\right)^{2}}\left[-Y^{0} \frac{\partial \Omega}{\partial Y^{0}}+T^{a} \frac{\partial \Omega}{\partial T^{a}}\right], \\
T^{a} & \rightarrow \frac{T^{a}}{\Delta_{\mathrm{T}}} .
\end{aligned}
$$

When the $\partial \Omega / \partial S$ term is suppressed in (3.17), one obtains the result,

$$
(T+\bar{T})^{a} \eta_{a b}(T+\bar{T})^{b} \rightarrow \frac{1}{\left|\Delta_{\mathrm{T}}\right|^{2}}(T+\bar{T})^{a} \eta_{a b}(T+\bar{T})^{b} .
$$

Assuming again that the above transformations constitute an invariance of the model, so that the T-duality transformation (3.16) of the $Y^{I}$ induces the expected transformations of the $F_{I}$ upon substitution, leads to

$$
\begin{aligned}
\left(\frac{\partial \Omega}{\partial S}\right)_{\mathrm{T}}^{\prime} & =\frac{\partial \Omega}{\partial S}, \\
\left(\frac{\partial \Omega}{\partial T^{a}}\right)_{\mathrm{T}}^{\prime} & =\left(\Delta_{\mathrm{T}} \delta_{a}{ }^{b}-2 \eta_{a c} T^{c} T^{b}\right) \frac{\partial \Omega}{\partial T^{b}}+2 \eta_{a b} T^{b} Y^{0} \frac{\partial \Omega}{\partial Y^{0}}, \\
\left(Y^{0} \frac{\partial \Omega}{\partial Y^{0}}\right)_{\mathrm{T}}^{\prime} & =Y^{0} \frac{\partial \Omega}{\partial Y^{0}}+\frac{4}{\Delta_{\mathrm{T}}\left(Y^{0}\right)^{2}} \frac{\partial \Omega}{\partial S}\left[-Y^{0} \frac{\partial \Omega}{\partial Y^{0}}+T^{a} \frac{\partial \Omega}{\partial T^{a}}\right] .
\end{aligned}
$$

This completes the review of the requirements for the function $\Omega$ derived in [2]. We stress once more that the central results, (3.15) and (3.20), hold in the presence of nonholomorphic modifications. Furthermore, it should be clear that $\Omega$ is not an invariant function. While the fields $\Upsilon$ and $\bar{\Upsilon}$ do not enter explicitly into the monodromies (3.4), (3.5) and (3.6), the corresponding transformations induced on $Y^{0}, S$, and $T^{a}$ depend in a complicated way on $\Upsilon$ and $\bar{\Upsilon}$.

\section{Performing the Legendre transform}

In this section we will now consider the new variables $\tilde{Y}^{I}$, and the corresponding set of variables consisting of $\tilde{Y}^{0}, \tilde{S}$ and $\tilde{T}^{a}$. To explicitly evaluate the equations that determine the new variables is a rather laborious task. They involve polynomials of fourth degree in the various fields which we shall subsequently solve by iteration. This iteration leads to infinite expansions, which in most cases we truncate at some order. The reader who is not primarily interested in these manipulations, may skip this section upon first reading. At the end of the section we also reconsider the S- and T-duality transformations in the two sets of variables.

We start by noting that, at zeroth order in the Weyl background, the new variables are equal to the original variables $S, T^{a}$ and $Y^{0}$, so that it is convenient to write

$$
\begin{aligned}
\tilde{Y}^{0} & =Y^{0}+\Delta Y^{0}, \\
\tilde{S} & =S+\Delta S, \\
\tilde{T}^{a} & =T^{a}+\Delta T^{a} .
\end{aligned}
$$


Here the changes $\Delta$ are induced by the Weyl background, which is encoded in the dependence on the field $\Upsilon$. Observe that the changes $\Delta$ will be non-holomorphic due to the reality property of the map (2.13), thus leading to a change of complex structure. This non-holomorphicity is thus not related to the fact that the function $\Omega$ is not necessarily harmonic. Using (2.13) and the explicit expressions (3.10) we derive the following six real equations for these changes,

$$
\begin{aligned}
\operatorname{Re}\left[\Delta Y^{0}\right] & =0, \\
\operatorname{Im}\left[Y^{0} \Delta S+S \Delta Y^{0}+\Delta S \Delta Y^{0}\right] & =0, \\
\operatorname{Im}\left[Y^{0} \Delta T^{a}+T^{a} \Delta Y^{0}+\Delta T^{a} \Delta Y^{0}\right] & =0,
\end{aligned}
$$

and

$$
\begin{gathered}
\operatorname{Re}\left[\left(Y^{0}+\Delta Y^{0}\right)(T+\Delta T)^{a} \eta_{a b}(T+\Delta T)^{b}-Y^{0} T^{a} \eta_{a b} T^{b}\right]= \\
\frac{1}{Y^{0}} \frac{\partial \Omega}{\partial S}+\frac{1}{\bar{Y}^{0}} \frac{\partial \Omega}{\partial \bar{S}} \\
\operatorname{Re}\left[\left(Y^{0}+\Delta Y^{0}\right)(S+\Delta S)(T+\Delta T)^{a}-Y^{0} S T^{a}\right]= \\
\frac{1}{2 Y^{0}} \frac{\partial \Omega}{\partial T_{a}}+\frac{1}{2 \bar{Y}^{0}} \frac{\partial \Omega}{\partial \bar{T}_{a}} \\
\operatorname{Im}\left[\left(Y^{0}+\Delta Y^{0}\right)(S+\Delta S)(T+\Delta T)^{a} \eta_{a b}(T+\Delta T)^{b}-Y^{0} S T^{a} \eta_{a b} T^{b}\right]= \\
-\mathrm{i}\left[-\frac{\partial \Omega}{\partial Y^{0}}+\frac{\partial \Omega}{\partial \bar{Y}^{0}}+\frac{S}{Y^{0}} \frac{\partial \Omega}{\partial S}-\frac{\bar{S}}{\bar{Y}^{0}} \frac{\partial \Omega}{\partial \bar{S}}+\frac{T^{a}}{Y^{0}} \frac{\partial \Omega}{\partial T^{a}}-\frac{\bar{T}^{a}}{\bar{Y}^{0}} \frac{\partial \Omega}{\partial \bar{T}^{a}}\right]
\end{gathered}
$$

where here and henceforth indices $a, b, \ldots$ are lowered and raised with $\eta_{a b}$ and its inverse $\eta^{a b}$. The left-hand side of these equations are polynomials of at most fourth degree in $\Delta S$, $\Delta T^{a}, \Delta Y^{0}$, and their complex conjugates. The equations (4.3) and (4.4) can conveniently be written as

$$
\begin{aligned}
\left(Y^{0}+\Delta Y^{0}\right) \Delta S-\left(\bar{Y}^{0}-\Delta Y^{0}\right) \Delta \bar{S} & =-(S+\bar{S}) \Delta Y^{0} \\
\left(Y^{0}+\Delta Y^{0}\right) \Delta T^{a}-\left(\bar{Y}^{0}-\Delta Y^{0}\right) \Delta \bar{T}^{a} & =-(T+\bar{T})^{a} \Delta Y^{0}
\end{aligned}
$$

The six equations can be solved by iteration which will lead to explicit power expansions in first-order derivatives of $\Omega$.

Before proceeding we note that it is convenient to write all the $\Omega$-dependent terms in the form of one real and one complex combination,

$$
\begin{aligned}
\mathcal{T}_{a} & =\frac{1}{Y^{0}} \frac{\partial \Omega}{\partial T^{a}}+\frac{1}{\bar{Y}^{0}} \frac{\partial \Omega}{\partial \bar{T}^{a}}, \\
\mathcal{U} & =\frac{\partial \Omega}{\partial Y^{0}}-\frac{\partial \Omega}{\partial \bar{Y}^{0}}-\frac{(S+\bar{S})}{Y^{0}} \frac{\partial \Omega}{\partial S}+\frac{(T+\bar{T})^{a}}{\bar{Y}^{0}} \frac{\partial \Omega}{\partial \bar{T}^{a}} .
\end{aligned}
$$

With these definitions, we first consider (4.6), which, with the help of (4.8), takes the form,

$$
(S+\bar{S}+\Delta S+\Delta \bar{S})\left(Y^{0}+\Delta Y^{0}\right) \Delta T^{a}+(T+\bar{T})^{a} \bar{Y}^{0} \Delta \bar{S}=\mathcal{T}^{a} .
$$


Likewise (4.5) can be written, again with the help of (4.8), as

$$
\begin{aligned}
& \left(Y^{0}+\Delta Y^{0}\right) \Delta T_{a}(2 T+2 \bar{T}+\Delta T+\Delta \bar{T})^{a}= \\
& \quad-\Delta Y^{0}(T+\bar{T})_{a}(T+\bar{T}+\Delta \bar{T})^{a}-2(S+\bar{S})^{-1}\left[\mathcal{U}+\overline{\mathcal{U}}-(T+\bar{T})^{a} \mathcal{T}_{a}\right] .
\end{aligned}
$$

Finally, (4.7) can be written in the form,

$$
\begin{aligned}
\left(Y^{0}+\right. & \left.\Delta Y^{0}\right) \Delta S\left(T^{2}-\bar{T}^{2}\right) \\
+ & \left(\bar{Y}^{0}-\Delta Y^{0}\right) \Delta \bar{T}^{a}\left[(2 T+\Delta T)_{a}(S+\Delta S)-(2 \bar{T}+\Delta \bar{T})_{a}(\bar{S}+\Delta \bar{S})\right] \\
& -\Delta Y^{0}(T+\bar{T})^{a}\left[S(T+\bar{T}+\Delta T)_{a}+\Delta S(2 T+\Delta T)_{a}\right]= \\
& 2(S+\bar{S})^{-1}\left[-S \mathcal{U}+\bar{S} \overline{\mathcal{U}}+\left(S T^{a}-\bar{S} \bar{T}^{a}\right) \mathcal{T}_{a}\right] .
\end{aligned}
$$

By taking suitable linear combinations we now write these equations in a form such that the first-order term is just proportional to either $\Delta S, \Delta T^{a}$, or $\Delta Y^{0}$. This will enable us to directly obtain the first-order results, whereas the higher-order ones will follow from iteration. The equation for $\Delta S$ is as follows,

$$
\begin{aligned}
Y^{0}(T+\bar{T})^{2} \Delta S= & 2 \overline{\mathcal{U}}+\Delta Y^{0} \Delta S(T+\bar{T})^{a}(T+\bar{T}+\Delta T)_{a} \\
& -\left(\bar{Y}^{0}-\Delta Y^{0}\right) \Delta \bar{T}^{a}\left[\Delta S(2 T+2 \bar{T}+\Delta T)_{a}-(S+\bar{S}+\Delta \bar{S}) \Delta \bar{T}_{a}\right] .
\end{aligned}
$$

For $\Delta T^{a}$, the expression takes the form,

$$
\begin{aligned}
Y^{0}(S+\bar{S}) & (T+\bar{T})^{2} \Delta T^{a}=(T+\bar{T})^{2} \mathcal{T}^{a}-2(T+\bar{T})^{a} \mathcal{U} \\
& +(T+\bar{T})^{a} \Delta Y^{0} \Delta \bar{S}(T+\bar{T})^{b}(T+\bar{T}+\Delta \bar{T})_{b} \\
& +(T+\bar{T})^{a}\left(Y^{0}+\Delta Y^{0}\right) \Delta T^{b}\left[\Delta \bar{S}(2 T+2 \bar{T}+\Delta \bar{T})_{b}-(S+\bar{S}+\Delta S) \Delta T_{b}\right] \\
& -\Delta T^{a}(T+\bar{T})^{2}\left[\left(Y^{0}+\Delta Y^{0}\right)(\Delta S+\Delta \bar{S})+\Delta Y^{0}(S+\bar{S})\right]
\end{aligned}
$$

and for $\Delta Y^{0}$, one obtains,

$$
\begin{aligned}
(S+\bar{S})(T+\bar{T})^{2} \Delta Y^{0}= & 2(\mathcal{U}-\overline{\mathcal{U}}) \\
& -\Delta Y^{0}\left[2(\Delta S+\Delta \bar{S})(T+\bar{T})^{2}+(T+\bar{T})^{a}\left(\Delta T_{a} \Delta S+\Delta \bar{T}_{a} \Delta \bar{S}\right)\right] \\
& +\left(\bar{Y}^{0}-\Delta Y^{0}\right) \Delta \bar{T}^{a}\left[\Delta S(2 T+2 \bar{T}+\Delta T)_{a}-(S+\bar{S}+\Delta \bar{S}) \Delta \bar{T}_{a}\right] \\
& -\left(Y^{0}+\Delta Y^{0}\right) \Delta T^{a}\left[\Delta \bar{S}(2 T+2 \bar{T}+\Delta \bar{T})_{a}-(S+\bar{S}+\Delta S) \Delta T_{a}\right] .
\end{aligned}
$$

These three equations constitute quartic polynomials in $\Delta S, \Delta T^{a}$ and $\Delta Y^{0}$, which can, in principle, be solved by iteration. Obviously, the full solution for $\Delta S, \Delta T^{a}$ and $\Delta Y^{0}$ will then take the form of an infinite series of products of the functions $\mathcal{U}$ and $\mathcal{T}_{a}$. To simplify the iteration to higher orders, it is convenient to use (4.10) and (4.11) once more for the higher-order terms of (4.13)-(4.15). We find, respectively,

$$
\begin{aligned}
Y^{0}(T+\bar{T})^{2} \Delta S= & 2\left\{\overline{\mathcal{U}}+\frac{\Delta S\left[\mathcal{U}+\overline{\mathcal{U}}-(T+\bar{T})^{a} \mathcal{T}_{a}\right]}{S+\bar{S}}\right\} \\
& +\Delta \bar{T}^{a}\left[\mathcal{T}_{a}-(T+\bar{T})_{a} Y^{0} \Delta S\right]
\end{aligned}
$$




$$
\begin{aligned}
Y^{0}(S+\bar{S})(T+\bar{T})^{2} \Delta T^{a}= & (T+\bar{T})^{2}\left[\mathcal{T}^{a}-\Delta T^{a}\left(Y^{0}+\bar{Y}^{0}\right) \Delta \bar{S}\right] \\
& -2(T+\bar{T})^{a}\left\{\mathcal{U}+\frac{\Delta \bar{S}\left[\mathcal{U}+\overline{\mathcal{U}}-(T+\bar{T})^{b} \mathcal{T}_{b}\right]}{S+\bar{S}}\right\} \\
& -(T+\bar{T})^{a} \Delta T^{b}\left[\mathcal{T}_{b}-(T+\bar{T})_{b} \bar{Y}^{0} \Delta \bar{S}\right], \\
(S+\bar{S})(T+\bar{T})^{2} \Delta Y^{0}=2 & \left\{\mathcal{U}-\overline{\mathcal{U}}+\frac{(\Delta \bar{S}-\Delta S)\left[\mathcal{U}+\overline{\mathcal{U}}-(T+\bar{T})^{a} \mathcal{T}_{a}\right]}{S+\bar{S}}\right\} \\
& +(\Delta T-\Delta \bar{T})^{a} \mathcal{T}_{a}-(T+\bar{T})^{2} \Delta Y^{0}(\Delta S+\Delta \bar{S}) \\
& -(T+\bar{T})^{a}\left[\bar{Y}^{0} \Delta T_{a} \Delta \bar{S}-Y^{0} \Delta \bar{T}_{a} \Delta S\right] .
\end{aligned}
$$

The lowest-order solution can be read off from (4.16)-(4.18), and takes the form,

$$
\begin{aligned}
Y^{0}(T+\bar{T})^{2} \Delta S & =2 \overline{\mathcal{U}} \\
Y^{0}(S+\bar{S})(T+\bar{T})^{2} \Delta T^{a} & =(T+\bar{T})^{2} \mathcal{T}^{a}-2(T+\bar{T})^{a} \mathcal{U}, \\
(S+\bar{S})(T+\bar{T})^{2} \Delta Y^{0} & =2(\mathcal{U}-\overline{\mathcal{U}})
\end{aligned}
$$

Resubstituting this result on the right-hand side of (4.16)-(4.18), yields the results to second order,

$$
\begin{aligned}
& Y^{0}(T+\bar{T})^{2} \Delta S \approx 2 \overline{\mathcal{U}}+\frac{4 \overline{\mathcal{U}}\left[\mathcal{U}+\overline{\mathcal{U}}-(T+\bar{T})^{a} \mathcal{T}_{a}\right]}{Y^{0}(S+\bar{S})(T+\bar{T})^{2}} \\
& +\frac{1}{\bar{Y}^{0}(S+\bar{S})}\left[\mathcal{T}^{a}-\frac{2(T+\bar{T})^{a} \overline{\mathcal{U}}}{(T+\bar{T})^{2}}\right]^{2}, \\
& Y^{0}(S+\bar{S})(T+\bar{T})^{2} \Delta T^{a} \approx(T+\bar{T})^{2} \mathcal{T}^{a}-2(T+\bar{T})^{a} \mathcal{U} \\
& -\frac{2\left(Y^{0}+\bar{Y}^{0}\right) \mathcal{U}\left[(T+\bar{T})^{2} \mathcal{T}^{a}-2(T+\bar{T})^{a} \mathcal{U}\right]}{\left|Y^{0}\right|^{2}(S+\bar{S})(T+\bar{T})^{2}} \\
& -4(T+\bar{T})^{a} \frac{\mathcal{U}\left[\mathcal{U}+\overline{\mathcal{U}}-(T+\bar{T})^{b} \mathcal{T}_{b}\right]}{\bar{Y}^{0}(S+\bar{S})(T+\bar{T})^{2}} \\
& -\frac{(T+\bar{T})^{a}}{Y^{0}(S+\bar{S})}\left[\mathcal{T}^{b}-\frac{2(T+\bar{T})^{b} \mathcal{U}}{(T+\bar{T})^{2}}\right]^{2}, \\
& (S+\bar{S})(T+\bar{T})^{2} \Delta Y^{0} \approx 2 \mathcal{U}+\frac{4 \mathcal{U}\left[2 \overline{\mathcal{U}}-(T+\bar{T})^{a} \mathcal{T}_{a}\right]}{\bar{Y}^{0}(S+\bar{S})(T+\bar{T})^{2}} \\
& +\frac{1}{Y^{0}(S+\bar{S})}\left[\mathcal{T}^{a}-\frac{2(T+\bar{T})^{a} \mathcal{U}}{(T+\bar{T})^{2}}\right]^{2} \\
& - \text { h.c. . }
\end{aligned}
$$

At the end of this section we briefly return to the transformation rules under S- and T-duality of the new variables. Obviously the previous equations should be consistent with these duality transformations. To verify this one first determines the transformation rules of $\Delta S, \Delta T^{a}$ and $\Delta Y^{0}$, which follow straightforwardly from their definition (4.1) and the 
transformations acting on the old and the new fields. In this way one obtains the following results under S-duality,

$$
\begin{aligned}
\Delta S & \rightarrow \frac{\Delta S}{\Delta_{\mathrm{S}}\left(\Delta_{\mathrm{S}}+\mathrm{i} c \Delta S\right)}, \\
\Delta T^{a} & \rightarrow \Delta T^{a}-\frac{\mathrm{i} c}{\Delta_{\mathrm{S}}\left(Y^{0}\right)^{2}} \frac{\partial \Omega}{\partial T_{a}} \\
\Delta Y^{0} & \rightarrow \frac{1}{2}\left(\Delta_{\mathrm{S}}+\bar{\Delta}_{\mathrm{S}}\right) \Delta Y^{0}+\frac{1}{2} \mathrm{i} c\left[\Delta S\left(Y^{0}+\Delta Y^{0}\right)+\Delta \bar{S}\left(\bar{Y}^{0}-\Delta Y^{0}\right)\right] .
\end{aligned}
$$

Likewise, under T-duality one finds,

$$
\begin{aligned}
\Delta S \rightarrow & \Delta S+\frac{2}{\Delta_{\mathrm{T}}\left(Y^{0}\right)^{2}}\left[Y^{0} \frac{\partial \Omega}{\partial Y^{0}}-T^{a} \frac{\partial \Omega}{\partial T^{a}}\right] \\
\Delta T^{a} \rightarrow & \frac{\Delta T^{a}}{(T+\Delta T)^{2}}+\frac{T^{a}}{(T+\Delta T)^{2} \Delta_{\mathrm{T}}}\left[-2 T^{b} \Delta T_{b}-(\Delta T)^{2}+\frac{2}{\left(Y^{0}\right)^{2}} \frac{\partial \Omega}{\partial S}\right], \\
\Delta Y^{0} \rightarrow & \frac{1}{2}\left(T^{2}+\bar{T}^{2}\right) \Delta Y^{0} \\
& +\frac{1}{2}\left[\left(Y^{0}+\Delta Y^{0}\right) \Delta T^{a}(2 T+\Delta T)_{a}-\left(\bar{Y}^{0}-\Delta Y^{0}\right) \Delta \bar{T}^{a}(2 \bar{T}+\Delta \bar{T})_{a}\right] \\
& -\left[\frac{1}{Y^{0}} \frac{\partial \Omega}{\partial S}-\frac{1}{\bar{Y}^{0}} \frac{\partial \Omega}{\partial \bar{S}}\right],
\end{aligned}
$$

where, in the T-duality transformation of $\Delta Y^{0}$, we made use of (4.5) in order to write the right-hand side in a form that is manifestly imaginary.

To verify the consistency we also need the transformations of the functions $\mathcal{U}$ and $\mathcal{T}^{a}$ under S- and T-duality, which follow from the results listed in section 3. Under S-duality these transformations take the following form,

$$
\begin{aligned}
\mathcal{T}_{a} & \rightarrow \frac{1}{\Delta_{\mathrm{S}} Y^{0}} \frac{\partial \Omega}{\partial T^{a}}+\frac{1}{\bar{\Delta}_{\mathrm{S}} \bar{Y}^{0}} \frac{\partial \Omega}{\partial \bar{T}^{a}}, \\
\mathcal{U} & \rightarrow \frac{\mathcal{U}}{\bar{\Delta}_{\mathrm{S}}}+\frac{\mathrm{i} c}{\left|\Delta_{\mathrm{S}}\right|^{2}\left(Y^{0}\right)^{2}} \mathcal{T}^{a} \frac{\partial \Omega}{\partial T^{a}}+\frac{c^{2}(S+\bar{S})}{2\left|\Delta_{\mathrm{S}}\right|^{2} \Delta_{\mathrm{S}}\left(Y^{0}\right)^{3}} \frac{\partial \Omega}{\partial T^{a}} \eta^{a b} \frac{\partial \Omega}{\partial T^{b}} .
\end{aligned}
$$

Under T-duality one derives the following transformations,

$$
\begin{aligned}
\mathcal{T}^{a} \rightarrow & \mathcal{T}^{a}+\frac{2 T^{a}}{\Delta_{\mathrm{T}}}\left(\frac{\partial \Omega}{\partial Y^{0}}-\frac{T^{b}}{Y^{0}} \frac{\partial \Omega}{\partial T^{b}}\right)+\frac{2 \bar{T}^{a}}{\bar{\Delta}_{\mathrm{T}}}\left(\frac{\partial \Omega}{\partial \bar{Y}^{0}}-\frac{\bar{T}^{b}}{\bar{Y}^{0}} \frac{\partial \Omega}{\partial \bar{T}^{b}}\right) \\
\mathcal{U} \rightarrow & \frac{\mathcal{U}}{\Delta_{\mathrm{T}}}+\frac{(T+\bar{T})^{2}}{\left|\Delta_{\mathrm{T}}\right|^{2}}\left(\frac{\partial \Omega}{\partial \bar{Y}^{0}}-\frac{\bar{T}^{a}}{\bar{Y}^{0}} \frac{\partial \Omega}{\partial \bar{T}^{a}}\right) \\
& -\frac{2}{\left(\Delta_{\mathrm{T}}\right)^{2}\left(Y^{0}\right)^{2}} \frac{\partial \Omega}{\partial S}\left(\frac{\partial \Omega}{\partial Y^{0}}-\frac{T^{a}}{Y^{0}} \frac{\partial \Omega}{\partial T^{a}}\right) \\
& +\frac{2}{\left|\Delta_{\mathrm{T}}\right|^{2}}\left(\frac{1}{\left(Y^{0}\right)^{2}} \frac{\partial \Omega}{\partial S}+\frac{1}{\left|Y^{0}\right|^{2}} \frac{\partial \Omega}{\partial S}+\frac{1}{\left(\bar{Y}^{0}\right)^{2}} \frac{\partial \Omega}{\partial \bar{S}}\right)\left(\frac{\partial \Omega}{\partial \bar{Y}^{0}}-\frac{\bar{T}^{a}}{\bar{Y}^{0}} \frac{\partial \Omega}{\partial \bar{T}^{a}}\right) .
\end{aligned}
$$

Here $\Delta_{\mathrm{S}}$ and $\Delta_{\mathrm{T}}$ were defined in (3.12) and (3.17). With the above results (4.23)-(4.26) one can verify that the equations (4.16)-(4.18) for $\Delta S, \Delta T^{a}$ and $\Delta Y^{0}$ are fully consistent with S- and T-duality. 


\section{$5 \quad$ Heterotic $N=4$ supersymmetric string compactifications}

As a first application we demonstrate the results of the previous section in a specific example, which is relevant in the context of $N=4$ supersymmetric models. Namely we assume that $\Omega$ depends only on $S$ and $\bar{S}$. Homogeneity then implies that $\Omega$ will depend linearly on $\Upsilon$ and its complex conjugate. In this case we have

$$
\mathcal{U}=-\frac{S+\bar{S}}{Y^{0}} \frac{\partial \Omega}{\partial S}, \quad \mathcal{T}_{a}=0 .
$$

By direct inspection it follows that the S-duality transformations take the simple form given by (3.7) and that the equations (3.15) are satisfied provided that $\Omega$ is an S-duality invariant function. For T-duality the situation is more subtle. The equations (3.20) are manifestly satisfied, but the transformation rules under T-duality take a complicated form,

$$
S \rightarrow S, \quad T^{a} \rightarrow \frac{T^{a}}{\Delta_{\mathrm{T}}}, \quad Y^{0} \rightarrow \Delta_{\mathrm{T}} Y^{0},
$$

where $\Delta_{\mathrm{T}}$ is still given by (3.17). Hence this example is consistent with both dualities. Under $\mathrm{S}$ - and T-duality $\mathcal{U}$ now transforms according to

$$
\mathcal{U} \rightarrow \frac{\mathcal{U}}{\bar{\Delta}_{\mathrm{S}}}, \quad \mathcal{U} \rightarrow \frac{\mathcal{U}}{\Delta_{\mathrm{T}}} .
$$

respectively.

As it turns out, it is convenient to introduce an S-duality invariant variable $\mathcal{V}$, defined by

$$
\mathcal{V}=-\frac{\mathcal{U}}{Y^{0}(S+\bar{S})(T+\bar{T})^{2}}=\frac{1}{\left(Y^{0}\right)^{2}(T+\bar{T})^{2}} \frac{\partial \Omega}{\partial S},
$$

so that the definition $(3.17)$ reads

$$
\Delta_{\mathrm{T}}=T^{2}+2(T+\bar{T})^{2} \mathcal{V}
$$

Under T-duality $\mathcal{V}$ transforms non-trivially according to

$$
\mathcal{V} \rightarrow \frac{\bar{\Delta}_{\mathrm{T}}}{\Delta_{\mathrm{T}}} \mathcal{V}\left[1+2(\mathcal{V}+\overline{\mathcal{V}})-2\left(\frac{\bar{\Delta}_{\mathrm{T}}}{\Delta_{\mathrm{T}}} \mathcal{V}+\frac{\Delta_{\mathrm{T}}}{\bar{\Delta}_{\mathrm{T}}} \overline{\mathcal{V}}\right)\right]^{-1}
$$

To solve the various equations of the previous section, we note that $\Delta T^{a}$ must be proportional to $(T+\bar{T})^{a}$ in this example. The proportionality factor turns out to be a function of the variable $\mathcal{V}$ and its complex conjugate, and is therefore S-duality invariant. The full expressions for $\Delta T^{a}, \Delta S$ and $\Delta Y^{0}$ then take the form,

$$
\begin{aligned}
\Delta T^{a} & =f(T+\bar{T})^{a}, \\
\Delta S & =-\frac{\bar{f}(S+\bar{S}) \bar{Y}^{0}}{(1+\bar{f}) Y^{0}+\bar{f} \bar{Y}^{0}}, \\
\Delta Y^{0} & =\frac{\bar{f} \bar{Y}^{0}-f Y^{0}}{1+f+\bar{f}},
\end{aligned}
$$


or, alternatively,

$$
\begin{aligned}
\tilde{T}^{a} & =(1+f) T^{a}+f \bar{T}^{a}, \\
\tilde{S} & =\frac{(1+\bar{f}) S Y^{0}-\bar{f} \bar{S} \bar{Y}^{0}}{(1+\bar{f}) Y^{0}+\bar{f} \bar{Y}^{0}}, \\
\tilde{Y}^{0} & =\frac{(1+\bar{f}) Y^{0}+\bar{f} \bar{Y}^{0}}{1+f+\bar{f}},
\end{aligned}
$$

where the function $f(\mathcal{V}, \overline{\mathcal{V}})$ is defined by a quadratic equation,

$$
|f|^{2}+f=2 \mathcal{V}
$$

From this result it follows that

$$
(1+f+\bar{f})^{2}=1+4(\mathcal{V}+\overline{\mathcal{V}})+4(\mathcal{V}-\overline{\mathcal{V}})^{2} \geq 0 .
$$

The solution for $f(\mathcal{V}, \overline{\mathcal{V}})$ reads as follows,

$$
f=-\frac{1}{2}+\mathcal{V}-\overline{\mathcal{V}} \pm \frac{1}{2} \sqrt{1+4(\mathcal{V}+\overline{\mathcal{V}})+4(\mathcal{V}-\overline{\mathcal{V}})^{2}}
$$

where one must adopt the plus sign in order to correctly reproduce the situation where $\Omega$ vanishes.

The new fields (5.8) transform indeed as required. With regard to T-duality the following transformation of $f$ under T-duality,

$$
f \rightarrow \frac{\bar{\Delta}_{\mathrm{T}}}{\Delta_{\mathrm{T}}} f\left[1+f-\frac{\bar{\Delta}_{\mathrm{T}}}{\Delta_{\mathrm{T}}} f\right]^{-1},
$$

is sufficient to ensure the correct T-duality transformations for the fields (5.8). Incidentally, (5.12) can be rewritten as

by making use of

$$
f \rightarrow \frac{\bar{\Delta}_{\mathrm{T}}}{\tilde{T}^{2}} f
$$

$$
\begin{aligned}
\tilde{T}^{2} & =(1+f) T^{2}-f \bar{T}^{2}+f(1+f)(T+\bar{T})^{2} \\
& =(1+f) \Delta_{\mathrm{T}}-f \bar{\Delta}_{\mathrm{T}} .
\end{aligned}
$$

Subsequently we evaluate the first term of the Hesse potential (2.12),

$$
\begin{aligned}
-\mathrm{i}\left(\bar{Y}^{I} F_{I}-Y^{I} \bar{F}_{I}\right) & =-\left|\tilde{Y}^{0}\right|^{2}(\tilde{S}+\overline{\tilde{S}})(\tilde{T}+\overline{\tilde{T}})^{2}\left[1+\frac{2|f|^{2}}{1+f+\bar{f}}\right] \\
& =-\left|\tilde{Y}^{0}\right|^{2}(\tilde{S}+\overline{\tilde{S}})(\tilde{T}+\overline{\tilde{T}})^{2} \frac{1+2(\mathcal{V}+\overline{\mathcal{V}})}{\sqrt{1+4(\mathcal{V}+\mathcal{V})+4(\mathcal{V}-\mathcal{V})^{2}}}
\end{aligned}
$$

where the sign adopted in the last expression is consistent with the sign choice noted below (5.11). To evaluate this result we used the following equations,

$$
\begin{aligned}
Y^{0} & =(1+f) \tilde{Y}^{0}-\bar{f} \overline{\tilde{Y}}^{0}, \\
(\tilde{T}+\overline{\tilde{T}})^{a} & =(1+f+\bar{f})(T+\bar{T})^{a}, \\
\tilde{S}+\overline{\tilde{S}} & =\frac{(1+f+\bar{f})\left|Y^{0}\right|^{2}(S+\bar{S})}{\left|(1+\bar{f}) Y^{0}+\bar{f} \bar{Y}^{0}\right|^{2}} .
\end{aligned}
$$


Subsequently it is convenient to introduce a quantity $\lambda$,

$$
\begin{aligned}
\lambda & =\frac{\bar{f}}{1+f} \frac{\overline{\tilde{Y}}^{0}}{\tilde{Y}^{0}} \\
& =-\frac{1+2(\mathcal{V}-\overline{\mathcal{V}})-\sqrt{1+4(\mathcal{V}+\overline{\mathcal{V}})+4(\mathcal{V}-\overline{\mathcal{V}})^{2}}}{1+2(\mathcal{V}-\overline{\mathcal{V}})+\sqrt{1+4(\mathcal{V}+\overline{\mathcal{V}})+4(\mathcal{V}-\overline{\mathcal{V}})^{2}}} \frac{\bar{Y}^{0}}{\tilde{Y}^{0}}
\end{aligned}
$$

so that

$$
\Delta S=-(\tilde{S}+\overline{\tilde{S}}) \frac{\lambda}{1-\lambda} .
$$

The newly defined quantity $\lambda$ is invariant under T-duality, while under S-duality it transforms with a phase factor,

$$
\lambda \rightarrow \frac{\overline{\tilde{\Delta}}_{\mathrm{S}}}{\tilde{\Delta}_{\mathrm{S}}} \lambda
$$

where $\tilde{\Delta}_{\mathrm{S}}=d+\mathrm{i} c \tilde{S}$.

We thus obtain the following expression for $\mathcal{V}$,

$$
\mathcal{V}=\left(\frac{1}{\tilde{Y}^{0}}+\frac{\lambda}{\overline{\tilde{Y}}^{0}}\right)^{2} \frac{1}{(\tilde{T}+\tilde{\tilde{T}})^{2}} \frac{1}{(1-\lambda)^{2}} \frac{\partial \Omega}{\partial S} .
$$

It remains to write the derivative of $\Omega(S, \bar{S})$ as a function of the new field $\tilde{S}$ and its complex conjugate. This can simply be done by writing $\Omega$ as

$$
\Omega(S, \bar{S})=\Omega\left(\tilde{S}+\frac{(\tilde{S}+\overline{\tilde{S}}) \lambda}{1-\lambda}, \overline{\tilde{S}}+\frac{(\tilde{S}+\overline{\tilde{S}}) \bar{\lambda}}{1-\bar{\lambda}}\right)
$$

and Taylor-expanding $(1-\lambda)^{-2} \partial_{S} \Omega$ in $\lambda$ and $\bar{\lambda}$. This leads to a double expansion in multiple covariant derivatives,

$$
\frac{\partial_{S} \Omega(S, \bar{S})}{(1-\lambda)^{2}}=\sum_{m=1, n=0}^{\infty} c_{(m, n)}(|\lambda|) \lambda^{m-1} \bar{\lambda}^{n}(\tilde{S}+\overline{\tilde{S}})^{m+n-1}\left(D_{\tilde{S}}\right)^{m}\left(\bar{D} \tilde{\bar{S}}^{n} \Omega(\tilde{S}, \tilde{\bar{S}}),\right.
$$

with respect to the new fields. Here the $c_{(m, n)}(\lambda)$ are functions of $|\lambda|$. The covariant derivatives are defined as follows. A modular form $\omega_{p, q}(S, \bar{S})$ of degree $(p, q)$ transforms according to $\omega_{p, q}(S, \bar{S}) \rightarrow[\Delta(S)]^{p}[\bar{\Delta}(\bar{S})]^{q} \omega_{p, q}(S, \bar{S})$. Its covariant derivative $D_{S}$ is then defined by

$$
D_{S} \omega_{p, q}(S, \bar{S})=\left(\partial_{S}+\frac{p}{S+\bar{S}}\right) \omega_{p, q}(S, \bar{S}),
$$

and transforms as $D_{S} \omega_{p, q}(S, \bar{S}) \rightarrow[\Delta(S)]^{p+2}[\bar{\Delta}(\bar{S})]^{q} D_{S} \omega_{p, q}(S, \bar{S})$. The covariant derivative with respect to $\bar{S}$ is defined likewise.

To obtain the Hesse potential (2.12), it remains to add $4 \Omega$ to (5.15). Using the same strategy as above, we can derive an expression similar to (5.22) for $\Omega$ expressed in $\tilde{S}$ and its complex conjugate,

$$
\Omega(S, \bar{S})=\sum_{m, n=0}^{\infty} d_{(m, n)}(|\lambda|) \lambda^{m} \bar{\lambda}^{n}(\tilde{S}+\overline{\tilde{S}})^{m+n}\left(D_{\tilde{S}}\right)^{m}\left(\bar{D}_{\tilde{\bar{S}}}\right)^{n} \Omega(\tilde{S}, \tilde{\bar{S}}) .
$$


As an illustration we have evaluated all contributions up to third order in $\Upsilon, \bar{\Upsilon}$. The first term in the Hesse potential (2.12), which in the case at hand is given by (5.15), can be expanded in powers of $\mathcal{V}$ and $\lambda$, making use of (5.17) and (5.20). In this way the result is expressed in terms of the new fields $\tilde{Y}^{0}, \tilde{S}$ and $\tilde{T}^{a}$. Up to third order one obtains (where on the right-hand side we have suppressed the tilde for clarity of notation),

$$
\begin{aligned}
-\mathrm{i}\left(\bar{Y}^{I} F_{I}-Y^{I} \bar{F}_{I}\right) \approx & -\left|Y^{0}\right|^{2}(S+\bar{S})(T+\bar{T})^{2} \\
& -\frac{8(S+\bar{S})\left|\partial_{S} \Omega\right|^{2}}{\left|Y^{0}\right|^{2}(T+\bar{T})^{2}}\left(1+\frac{4(S+\bar{S}) \partial_{S} \partial_{\bar{S}} \Omega}{\left|Y^{0}\right|^{2}(T+\bar{T})^{2}}\right) \\
& -\frac{16(S+\bar{S})^{2}}{\left|Y^{0}\right|^{4}\left[(T+\bar{T})^{2}\right]^{2}}\left(\left(\partial_{S} \Omega\right)^{2} \bar{D}_{\bar{S}} \partial_{\bar{S}} \Omega+\left(\partial_{\bar{S}} \Omega\right)^{2} D_{S} \partial_{S} \Omega\right) .
\end{aligned}
$$

This expression is manifestly invariant under both S- and T-duality. Subsequently we evaluate (5.24), and obtain the following result, after again suppressing the tildes on the right-hand side,

$$
\begin{aligned}
\Omega(S, \bar{S}) \approx & \Omega(S, \bar{S}) \\
& +\frac{4(S+\bar{S})\left|\partial_{S} \Omega\right|^{2}}{\left|Y^{0}\right|^{2}(T+\bar{T})^{2}}\left(1+\frac{3(S+\bar{S})}{\left|Y^{0}\right|^{2}(T+\bar{T})^{2}} \partial_{S} \partial_{\bar{S}} \Omega\right) \\
& +\frac{6(S+\bar{S})^{2}}{\left|Y^{0}\right|^{4}\left[(T+\bar{T})^{2}\right]^{2}}\left(\left(\partial_{\bar{S}} \Omega\right)^{2} D_{S} \partial_{S} \Omega+\left(\partial_{S} \Omega\right)^{2} \bar{D}_{\bar{S}} \partial_{\bar{S}} \Omega\right)
\end{aligned}
$$

This is manifestly invariant under S- and T-duality. We observe that both (5.25) and (5.26) depend non-holomorphically on $\tilde{Y}^{0}$ (we reinstate the tilde to indicate that we are discussing the new variables). The only exception is the first term in (5.26), which is equal to $\Omega$ and does not depend on $\tilde{Y}^{0}$, nor on its complex conjugate. All the terms arising in higher orders will always depend on $\left|\tilde{Y}^{0}\right|$, and not on $\tilde{Y}^{0}$ or $\tilde{Y}^{0}$, separately. This is because invariance under T-duality dictates that each power of $(\tilde{T}+\overline{\tilde{T}})^{2}$ (arising by power expanding in $\mathcal{V}$ ) has to appear multiplied by $\left|\tilde{Y}^{0}\right|^{2}$. We return to the significance of this observation in later sections.

\section{The Hesse potential at second order}

In this section, we return to the general case based on (3.10) and we consider the Hesse potential, using the representation (2.12). It consists of two parts which both transform as proper functions under electric/magnetic duality. The first term is equal to

$$
\begin{aligned}
-\mathrm{i}\left(\bar{Y}^{I} F_{I}-Y^{I} \bar{F}_{I}\right)= & -\left|Y^{0}\right|^{2}(S+\bar{S})(T+\bar{T})^{2}+2\left(Y^{0} \frac{\partial \Omega}{\partial Y^{0}}+\bar{Y}^{0} \frac{\partial \Omega}{\partial \bar{Y}^{0}}\right) \\
& +2\left(\bar{Y}^{0} \mathcal{U}+Y^{0} \overline{\mathcal{U}}\right)-2\left(Y^{0}+\bar{Y}^{0}\right)(T+\bar{T})^{a} \mathcal{T}_{a},
\end{aligned}
$$

where we made use of (3.10). The second term contributing to the Hesse potential is equal to $4\left(\Upsilon \partial_{\Upsilon} \Omega+\bar{\Upsilon} \partial_{\bar{\Upsilon}} \Omega\right)$. This term is separately invariant under the dualities. Combining both terms and making use of the homogeneity of the function $\Omega$, i.e., $2 \Omega=2 \Upsilon \Omega_{\Upsilon}+$ 
$2 \bar{\Upsilon} \Omega_{\bar{\Upsilon}}+Y^{I} \Omega_{I}+\bar{Y}^{I} \Omega_{\bar{I}}$, it follows that the Hesse potential takes the form,

$$
\begin{aligned}
\mathcal{H}(\tilde{Y}, \overline{\tilde{Y}}, \Upsilon, \bar{\Upsilon})= & -\left|Y^{0}\right|^{2}(S+\bar{S})(T+\bar{T})^{2}+4 \Omega\left(Y^{0}, \bar{Y} 0, S, \bar{S}, T, \bar{T}, \Upsilon, \bar{\Upsilon}\right) \\
& +2\left(\bar{Y}^{0} \mathcal{U}+Y^{0} \overline{\mathcal{U}}\right)-2\left(Y^{0}+\bar{Y}^{0}\right)(T+\bar{T})^{a} \mathcal{T}_{a} .
\end{aligned}
$$

This result is written as a function of the old fields $Y^{0}, S$, and $T^{a}$, which can be expressed in terms of the new fields by using (4.1). Expressing the first term in the new fields $\tilde{Y}^{0}$, $\tilde{S}$ and $\tilde{T}^{a}$, generates contributions up to fifth order in $\Delta Y^{0}, \Delta S$ and $\Delta T^{a}$, which, upon iteration, can be expressed as a power series in $\mathcal{U}$ and $\mathcal{T}_{a}$. Here we will consider terms of first- and second-order. As it turns out, the first-order term cancel against those explicitly given in (6.1). This is a general phenomenon which applies also to other models than the ones based on (3.1). Making use of (4.20), (4.21) and (4.22), we obtain,

$$
\begin{aligned}
\mathcal{H}(\tilde{Y}, \overline{\tilde{Y}}, \Upsilon, \bar{\Upsilon}) \approx & -\left|\tilde{Y}^{0}\right|^{2}(\tilde{S}+\overline{\tilde{S}})(\tilde{T}+\overline{\tilde{T}})^{2}+4 \Omega\left(Y^{0}, \bar{Y}{ }^{0}, S, \bar{S}, T, \bar{T}, \Upsilon, \bar{\Upsilon}\right) \\
& +\frac{2 \mathcal{T}_{a} \eta^{a b} \mathcal{T}_{b}}{S+\bar{S}}-\frac{8|\mathcal{U}|^{2}}{(S+\bar{S})(T+\bar{T})^{2}}+\cdots,
\end{aligned}
$$

where the ellipses denote terms of third and higher order in derivatives of $\Omega$. In this approximation the result is invariant under S- and T-duality, as it should. The first term is manifestly invariant. The invariance of the remaining three terms follows directly from application of the relevant equations in (3.15), (3.20), (4.25) and (4.26).

Subsequently we express $\Omega$ in terms of the new variables. This can be done by Taylor expanding, using (4.1),

$$
\begin{gathered}
\Omega\left(Y^{0}, \bar{Y}^{0}, S, \bar{S}, T, \bar{T}\right) \approx \Omega\left(\tilde{Y}^{0}, \overline{\tilde{Y}}^{0}, \tilde{S}, \overline{\tilde{S}}, \tilde{T}, \overline{\tilde{T}}\right)-\left.\Delta Y^{0}\left(\frac{\partial \Omega}{\partial Y^{0}}-\frac{\partial \Omega}{\partial \bar{Y}^{0}}\right)\right|_{*} \\
-\left.\Delta S \frac{\partial \Omega}{\partial S}\right|_{*}-\left.\Delta \bar{S} \frac{\partial \Omega}{\partial \bar{S}}\right|_{*}-\left.\Delta T^{a} \frac{\partial \Omega}{\partial T^{a}}\right|_{*}-\left.\Delta \bar{T}^{a} \frac{\partial \Omega}{\partial \bar{T}^{a}}\right|_{*}+\cdots,
\end{gathered}
$$

where we have suppressed the variables $\Upsilon$. The notation $\left.\right|_{*}$ indicates that the derivatives are taken at $\tilde{Y}^{0}, \tilde{S}$ and $\tilde{T}$. However, in the approximation that we adopted, this aspect is only relevant in higher orders. The terms generated by the Taylor expansion turn out to be proportional to $|\mathcal{U}|^{2}$ and $\mathcal{T}_{a} \eta^{a b} \mathcal{T}_{b}$. Substituting the above result into (6.3), we obtain

$$
\begin{aligned}
\mathcal{H}(\tilde{Y}, \overline{\tilde{Y}}, \Upsilon, \bar{\Upsilon}) \approx & -\left|\tilde{Y}^{0}\right|^{2}(\tilde{S}+\overline{\tilde{S}})(\tilde{T}+\overline{\tilde{T}})^{2}+4 \Omega\left(\tilde{Y}^{0}, \overline{\tilde{Y}} 0, \tilde{S}, \overline{\tilde{S}}, \tilde{T}, \overline{\tilde{T}}\right) \\
& -\frac{2 \mathcal{T}_{a} \eta^{a b} \mathcal{T}_{b}}{S+\bar{S}}+\frac{8|\mathcal{U}|^{2}}{(S+\bar{S})(T+\bar{T})^{2}},
\end{aligned}
$$

which holds to second order in $\Omega$ and derivatives thereof.

In order to compare with other results we write $\Omega$ as a power series in the Weyl background $\Upsilon$ (just as in (3.2)) and/or $\bar{\Upsilon}$, so that we may write,

$$
\Omega\left(Y^{0}, \bar{Y}^{0}, S, \bar{S}, T, \bar{T}, \Upsilon, \bar{\Upsilon}\right)=\sum_{g=1}^{\infty} \Omega^{(g)}\left(Y^{0}, \bar{Y}^{0}, S, \bar{S}, T, \bar{T}\right),
$$

where $\Omega^{(g)}$ is real and decomposable in monomials of the form $\Upsilon^{n} \bar{\Upsilon}^{g-n}$, with $0 \leq n \leq g$. For conciseness, we will refrain from explicitly indicating the dependence of the functions 
$\Omega^{(g)}$ on $\Upsilon$ and $\bar{\Upsilon}$. In the approximation that we retain terms of second order of $\Upsilon$, $\bar{\Upsilon}$, we will only have contributions from $\Omega^{(1)}$ and $\Omega^{(2)}$ which we can regard as functions of the new fields $\tilde{Y}^{I}$. Since the result depends then only on the new fields $\tilde{Y}^{I}$, we can now consistently drop the distinction between the variables $\tilde{Y}^{I}$ and $Y^{I}$ to simplify our notation. Therefore, we suppress the tilde on the right-hand side in the formula below,

$$
\begin{aligned}
\mathcal{H}(\tilde{Y}, \overline{\tilde{Y}}, \Upsilon, \bar{\Upsilon}) \approx & -\left|Y^{0}\right|^{2}(S+\bar{S})(T+\bar{T})^{2}+4 \Omega^{(1)}(S, \bar{S}, T, \bar{T}) \\
& +4 \Omega^{(2)}\left(Y^{0}, \bar{Y}^{0}, S, \bar{S}, T, \bar{T}\right) \\
& -\left\{\frac{2}{\left(Y^{0}\right)^{2}}\left(\frac{\partial \Omega^{(1)}}{\partial T^{a}}\left[\frac{1}{S+\bar{S}} \frac{\partial \Omega^{(1)}}{\partial T_{a}}+\frac{4(T+\bar{T})_{a}}{(T+\bar{T})^{2}} \frac{\partial \Omega^{(1)}}{\partial S}\right]\right)+\text { h.c. }\right\} \\
& +\frac{4(T+\bar{T})^{a}(T+\bar{T})^{b}}{\left|Y^{0}\right|^{2}(S+\bar{S})(T+\bar{T})^{2}}\left(2 \frac{\partial \Omega^{(1)}}{\partial T^{a}} \frac{\partial \Omega^{(1)}}{\partial \bar{T}^{b}}-\eta_{a b} \frac{\partial \Omega^{(1)}}{\partial T_{c}} \frac{\partial \Omega^{(1)}}{\partial \bar{T}^{c}}\right) \\
& +\frac{8(S+\bar{S})}{\left|Y^{0}\right|^{2}(T+\bar{T})^{2}} \frac{\partial \Omega^{(1)}}{\partial S} \frac{\partial \Omega^{(1)}}{\partial \bar{S}}
\end{aligned}
$$

Note that $\Omega^{(1)}$ does not depend on $Y^{0}$ and $\bar{Y}^{0}$ because of homogeneity. The above result represents the Hesse potential to second order in $\Upsilon, \bar{\Upsilon}$ and is the basis for our discussion in the next section. Higher-order terms have a similar characteristics as the terms we derived in section 5. They always consist of the product of a number of first-order derivatives of $\Omega$, times a number of higher-order derivatives of $\Omega$.

Let us comment on the various terms in (6.7) (we again reinstate the tildes to stress that we are dealing with the new variables). First we note that $\Omega^{(1)}$ and $\Omega^{(2)}$ define the higher-derivative corrections to the effective action, but their arguments are not the moduli defined in that perspective, as they are based on the Legendre transform. By construction the expression should be duality invariant where the transformation rules are the ones that pertain to the classical action (i.e. without higher-derivative couplings), specified in (3.7) and (3.8).

The first $\Omega$-independent term in (6.7) is duality invariant and so is $\Omega^{(1)}$ (provided we take $\Upsilon$ real). This result applies to all orders, because of the new variables that have been employed. It is known that $\Omega^{(2)}$ is not duality invariant [2], and neither are the terms proportional to $\left(\tilde{Y}^{0}\right)^{-2}$ or $\left(\bar{Y}^{0}\right)^{-2}$. On the other hand the two terms proportional to $\left|\tilde{Y}^{0}\right|^{-2}$ are both S- and T-duality invariant. The relevance of this decomposition will be explained in the next section.

As we emphasized already in section 1 , we concentrate on S- and T-duality here in view of the fact that we have only explicit information about models with a high degree of symmetry. The decomposition in terms of $\left(\tilde{Y}^{0}\right)^{-2},\left(\bar{Y}^{0}\right)^{-2}$ and $\left|\tilde{Y}^{0}\right|^{-2}$ remains relevant in the more general case, as $\tilde{Y}^{0}$ is the inverse holomorphic coupling constant of the topological string. Consequently, the terms proportional to $\left|\tilde{Y}^{0}\right|^{-2}$ cannot be part of the twisted partition function of genus $g=2$, irrespective of whether the model has certain duality invariances. 


\section{The Hesse potential for specific models}

In this section we consider the consequences of the results of the previous sections in the context of a few specific models. As was already mentioned in section 1, there are only a few models for which explicit results have been obtained for the effective action and/or the topological string. The models that we discuss are models with $N=4$ supersymmetry, cast in an $N=2$ description, and the FHSV model [5] (another possible model is the STU model $[6,7]$, but this is qualitatively similar to the FHSV model). As it turns out the expression for $\Omega^{(1)}(S, \bar{S}, T, \bar{T})$ coincides for both the effective action, the Hesse potential and the topological string.

We begin with the $N=4$ supersymmetric model discussed in section 5 for which $\Omega=$ $\Omega(S, \bar{S})$ depends only on $S$ and $\bar{S}$. Particular examples are the so-called CHL models [31]. Using (5.15), we obtain the following exact expression for the Hesse potential,

$$
\begin{aligned}
\mathcal{H}(\tilde{Y}, \overline{\tilde{Y}}, \Upsilon, \bar{\Upsilon})= & -\left|\tilde{Y}^{0}\right|^{2}(\tilde{S}+\overline{\tilde{S}})(\tilde{T}+\overline{\tilde{T}})^{2} \frac{1+2(\mathcal{V}+\overline{\mathcal{V}})}{\sqrt{1+4(\mathcal{V}+\mathcal{V})+4(\mathcal{V}-\overline{\mathcal{V}})^{2}}} \\
& +4 \Omega(S, \bar{S})
\end{aligned}
$$

Note that we have not constrained $\Omega(S, \bar{S})$ other than that it should be invariant under the S-duality group. The definition of $\mathcal{V}$ is subtle and follows from (5.20) and (5.17). The result was evaluated up to terms cubic in $\Omega$-derivatives, and combining the explicit results (5.25) and (5.26), one obtains the following expression (where, again, we suppressed the tildes on the right-hand side of the equation),

$$
\begin{aligned}
\mathcal{H}(\tilde{Y}, \overline{\tilde{Y}}, \Upsilon, \bar{\Upsilon}) \approx & -\left|Y^{0}\right|^{2}(S+\bar{S})(T+\bar{T})^{2}+4 \Omega(S, \bar{S}) \\
& +\frac{8(S+\bar{S})\left|\partial_{S} \Omega\right|^{2}}{\left|Y^{0}\right|^{2}(T+\bar{T})^{2}}\left(1+\frac{2(S+\bar{S}) \partial_{S} \partial_{\bar{S}} \Omega}{\left|Y^{0}\right|^{2}(T+\bar{T})^{2}}\right) \\
& +\frac{8(S+\bar{S})^{2}}{\left|Y^{0}\right|^{4}\left[(T+\bar{T})^{2}\right]^{2}}\left(\left(\partial_{S} \Omega\right)^{2} \bar{D}_{\bar{S}} \partial_{\bar{S}} \Omega+\left(\partial_{\bar{S}} \Omega\right)^{2} D_{S} \partial_{S} \Omega\right)
\end{aligned}
$$

to third order in $\Omega$ and derivatives thereof. This expression exhibits the dependence of the Hesse potential on both $\tilde{Y}^{0}$ and its complex conjugate (here we again reinstated the tilde). As we already argued in section 5 , the higher-order terms will depend only on $\left|\tilde{Y}^{0}\right|$, and no longer on $\tilde{Y}^{0}$ and $\overline{\tilde{Y}}^{0}$ separately.

For completeness we recall the expression for $\Omega_{k}$ for the CHL models distinguished by an integer label $k$. As discussed in [15] the function $\Omega_{k}$ can be expressed in terms of the unique cusp forms of weight $k+2$ associated with the S-duality group $\Gamma_{1}(\tilde{N}) \subset \mathrm{SL}(2 ; \mathbb{Z})$, defined by $f^{(k)}(S)=\eta^{k+2}(S) \eta^{k+2}(\tilde{N} S)$ where,

$$
f^{(k)}\left(S^{\prime}\right)=\Delta_{\mathrm{S}}^{k+2} f^{(k)}(S) .
$$

The result for $\Omega_{k}$ then takes the following form [1],

$$
\Omega_{k}(S, \bar{S}, \Upsilon, \bar{\Upsilon})=\frac{1}{256 \pi}\left[\Upsilon \ln f^{(k)}(S)+\bar{\Upsilon} \ln f^{(k)}(\bar{S})+\frac{1}{2}(\Upsilon+\bar{\Upsilon}) \ln (S+\bar{S})^{k+2}\right]
$$


Note that this result agrees with the terms obtained for the corresponding effective actions (see, for instance, $[13,32]$ ). These models are invariant under the S-duality group $\Gamma_{1}(\tilde{N}) \subset$ $\mathrm{SL}(2 ; \mathbb{Z})$, which is generated by $(3.13)$ with the transformation parameters restricted to $c=0 \bmod \tilde{N}$ and $a, d=1 \bmod \tilde{N}$.

Next, we consider the $N=2$ supersymmetric FHSV model. Its type-II realization corresponds to the compactification on the Enriques Calabi-Yau three-fold, which is described as an orbifold $\left(\mathrm{T}^{2} \times \mathrm{K} 3\right) / \mathbb{Z}_{2}$, where $\mathbb{Z}_{2}$ is a freely acting involution. The massless sector of the four-dimensional theory comprises 11 vector supermultiplets, 12 hypermultiplets and the $N=2$ graviton supermultiplet. The classical moduli space of the the vector multiplet sector equals the special-Kähler space,

$$
\mathcal{M}_{\text {vector }}=\frac{\mathrm{SL}(2)}{\mathrm{SO}(2)} \times \frac{\mathrm{O}(10,2)}{\mathrm{O}(10) \times \mathrm{O}(2)} .
$$

Its two factors are associated with $\mathrm{T}^{2} / \mathbb{Z}_{2}$ and the $\mathrm{K} 3$ fiber, and the special coordinates for these two spaces will be denoted by $S$ and $T^{a}$, respectively.

At first order in the Weyl background, the solution to (3.15) and (3.20) for the FHSV model is known from threshold corrections and from the topological string side [33, 34]. It takes the form [2],

$$
\begin{aligned}
\Omega^{(1)}(S, \bar{S}, T, \bar{T}, \Upsilon, \bar{\Upsilon})=\frac{1}{256 \pi} & {\left[\frac{1}{2} \Upsilon \ln \left[\eta^{24}(2 S) \Phi(T)\right]+\frac{1}{2} \bar{\Upsilon} \ln \left[\eta^{24}(2 \bar{S}) \Phi(\bar{T})\right]\right.} \\
& \left.+(\Upsilon+\bar{\Upsilon}) \ln \left[(S+\bar{S})^{3}(T+\bar{T})^{a} \eta_{a b}(T+\bar{T})^{b}\right]\right]
\end{aligned}
$$

For real values of $\Upsilon$, this result is invariant under S-duality, which constitute the $\Gamma(2)$ subgroup of $\mathrm{SL}(2 ; \mathbb{Z})$, defined by $a, d=1 \bmod 2$ and $b, c=0 \bmod 2$ in (3.7). The result is also invariant under the T-duality group $\mathrm{O}(10,2 ; \mathbb{Z})$ in view of the fact that $\Phi(T)$ is a holomorphic automorphic form of weight 4 [35], transforming under the T-duality transformation $T^{a} \rightarrow T^{a}\left[T^{2}\right]^{-1}$ as

$$
\Phi(T) \rightarrow\left[T^{2}\right]^{4} \Phi(T)
$$

Clearly, (7.6) can be written as the sum of two invariant functions, one of $S$ and $\bar{S}$ and one of $T^{a}$ and $\bar{T}^{a}$, respectively, which both contain non-holomorphic terms that are crucial for the duality invariance. Observe that the duality invariance of $\Omega^{(1)}$ is only realized for real values of $\Upsilon$. Therefore we do not know a priori whether to write $\Upsilon$ or its complex conjugate. The way in which this potential ambiguity has been resolved, is by assuming that purely holomorphic terms are always accompanied by a power of $\Upsilon$ and purely antiholomorphic terms by a power of $\bar{\Upsilon}$, whereas for the mixed terms we assign $\Upsilon$ and $\bar{\Upsilon}$ such as to preserve the reality properties of $\Omega$ for complex $\Upsilon$.

At second order in the Weyl background, the solution to (3.15) and (3.20) for the FHSV model takes the following form [2], up to an S- and T-duality invariant function,

$$
\Omega^{(2)}=-\frac{G_{2}(2 S)}{\left(Y^{0}\right)^{2}} \frac{\partial \Omega^{(1)}}{\partial T^{a}} \frac{\partial \Omega^{(1)}}{\partial T_{a}}-\frac{1}{4\left(Y^{0}\right)^{2}} \frac{\partial \ln \Phi(T)}{\partial T_{a}} \frac{\partial \Omega^{(1)}}{\partial T^{a}} \frac{\partial \Omega^{(1)}}{\partial S}+\text { c.c },
$$


where $G_{2}(2 S)=\frac{1}{2} \partial_{S} \ln \eta^{2}(2 S)$. Observe that $\Omega^{(2)}$ is not duality invariant. Inserting it into (6.7), one obtains (the right-hand side is expressed exclusively in terms of the new variables but we again suppress the tilde on the right-hand side for clarity),

$$
\begin{aligned}
\mathcal{H}(\tilde{Y}, \overline{\tilde{Y}}, \Upsilon, \bar{\Upsilon}) \approx & -\left|Y^{0}\right|^{2}(S+\bar{S})(T+\bar{T})^{2}+4 \Omega^{(1)}(S, \bar{S}, T, \bar{T}) \\
- & {\left[\frac{4 \hat{G}_{2}(2 S, 2 \bar{S})}{\left(Y^{0}\right)^{2}} \frac{\partial \Omega^{(1)}}{\partial T_{a}} \frac{\partial \Omega^{(1)}}{\partial T^{a}}\right.} \\
& \left.\quad+\frac{1}{\left(Y^{0}\right)^{2}} \frac{\partial \log \left[\Phi(T)\left[(T+\bar{T})^{2}\right]^{4}\right]}{\partial T_{a}} \frac{\partial \Omega^{(1)}}{\partial T^{a}} \frac{\partial \Omega^{(1)}}{\partial S}+\text { c.c. }\right] \\
+ & \frac{4(T+\bar{T})^{a}(T+\bar{T})^{b}}{\left|Y^{0}\right|^{2}(S+\bar{S})(T+\bar{T})^{2}}\left(2 \frac{\partial \Omega^{(1)}}{\partial T^{a}} \frac{\partial \Omega^{(1)}}{\partial \bar{T}^{b}}-\eta_{a b} \frac{\partial \Omega^{(1)}}{\partial T_{c}} \frac{\partial \Omega^{(1)}}{\partial \bar{T}^{c}}\right) \\
+ & \frac{8(S+\bar{S})}{\left|Y^{0}\right|^{2}(T+\bar{T})^{2}} \frac{\partial \Omega^{(1)}}{\partial S} \frac{\partial \Omega^{(1)}}{\partial \bar{S}},
\end{aligned}
$$

where $\hat{G}_{2}(2 S, 2 \bar{S})=G_{2}(2 S)+[2(S+\bar{S})]^{-1}$. In this expression, $\Omega^{(1)}$ is given by (7.6), with the old variables replaced by the new ones. This result is manifestly invariant under S- and T-duality, as it should. Furthermore, the terms proportional to $\left(Y^{0}\right)^{-2}$ can be combined by noting that, for real values of $\Upsilon$, we have the identities,

$$
\begin{aligned}
\frac{\partial \Omega^{(1)}}{\partial S} & =\frac{24 \Upsilon}{512 \pi} \hat{G}_{2}(2 S, 2 \bar{S}), \\
\frac{\partial \Omega^{(1)}}{\partial T^{a}} & =\frac{\Upsilon}{512 \pi} \frac{\partial \log \left[\Phi(T)\left[(T+\bar{T})^{2}\right]^{4}\right]}{\partial T^{a}} .
\end{aligned}
$$

Therefore these terms can be rewritten as (suppressing the tildes on both sides),

$$
\left.\mathcal{H}(Y, \bar{Y}, \Upsilon, \bar{\Upsilon})\right|_{\left(Y^{0}\right)^{-2}}=\frac{28 \hat{G}_{2}(2 S, 2 \bar{S})}{\left(Y^{0}\right)^{2}} \frac{\partial \Omega^{(1)}}{\partial T_{a}} \frac{\partial \Omega^{(1)}}{\partial T^{a}} .
$$

This is consistent with the result found for the topological string [8], apart from the overall normalization. However, we can change the normalization by including the same duality invariant expression into $\Omega^{(2)}$ with a different coefficient. Since (7.8) has only been determined up to a duality invariant function, and since we have no independent knowledge of the invariant parts in $\Omega^{(2)}$, the overall normalization given in (7.11) is therefore ambiguous. At this point we should recall that the topological string partition functions are derived from integrating the holomorphic anomaly equations [8], so that the results are in principle determined up to holomorphic contributions. On the other hand, results such as (7.8) have been obtained from requiring covariance under duality transformations, and therefore they determine the $\Omega^{(g)}$ up to duality invariant terms. Usually the invariant terms are non-holomorphic, so that combining the two methods could potentially remove the ambiguities. However, there can also be holomorphic, invariant functions, which would be missed in both approaches. As in [8], one may be able to remove some of these ambiguities by making use of knowledge of the boundary behaviour or certain asymptotic conditions, but at present this is not really known. 
Based on the previous arguments, and modulo the ambiguity noted above in the normalization of the genus- 2 contribution, the topological string partition function would correspond to the following function $F^{\text {top }}$, which can be viewed as the analogue of (2.2) for the effective action,

$$
\begin{aligned}
F^{\mathrm{top}} \approx & \mathrm{i}\left(Y^{0}\right)^{2} S T^{2}-\frac{\mathrm{i}}{2 \pi} \ln \left[\left|\eta^{24}(2 S)\right|(S+\bar{S})^{6}|\Phi(T)|\left[(T+\bar{T})^{2}\right]^{2}\right] \\
& +a \frac{\mathrm{i} \hat{G}_{2}(2 S, 2 \bar{S})}{\left(2 \pi Y^{0}\right)^{2}} \frac{\partial \ln \left[|\Phi(T)|\left[(T+\bar{T})^{2}\right]^{2}\right]}{\partial T_{a}} \frac{\partial \ln \left[|\Phi(T)|\left[(T+\bar{T})^{2}\right]^{2}\right]}{\partial T^{a}}
\end{aligned}
$$

where we set $\Upsilon=-64$. Here the real constant $a$ represents the ambiguity discussed above, which can be fixed by imposing the holomorphic anomaly equation. We stress that the arguments used in (7.12) refer to the new variables $\tilde{Y}^{0}, \tilde{S}$ and $\tilde{T}^{a}$, and that the topological string coupling constant $g_{\mathrm{s}}$ is inversely proportional to $\tilde{Y}^{0}$.

One may now wonder what the role is of the contributions to the Hesse potential that do not depend holomorphically on $\tilde{Y}^{0}$. These are the terms in (7.9) proportional to $\left|\tilde{Y}^{0}\right|^{-2}$, which are not part of the topological string partition function, as they do not depend holomorphically on the topological string coupling. Here we reinstated the tilde to emphasize that we are dealing with the new variables. Obviously these terms are duality invariant, and therefore their normalization factors can in principle be changed upon including similar invariant terms into $\Omega^{(2)}$. Hence the normalization remains ambiguous, which makes it hard to assess the relevance of these terms.

At this point let us return to the $N=4$ supersymmetric models and consider the expression for the Hesse potential (7.2). In this case there is only a dependence on $\left|\tilde{Y}^{0}\right|$ and the only contribution to the topological string originates from $\Omega(\tilde{S}, \overline{\tilde{S}})$. This result is in agreement with known results for the topological string partition function [3]. The normalization of this genus- 1 term is unambiguous, which lends support to the discussion of the FHSV model given above. Concerning the terms that depend on negative powers of $\left|\tilde{Y}^{0}\right|$, in principle such duality invariant terms can also be present in $\Omega$ as contributions to the effective action, and their presence would affect the normalization factors of the corresponding terms in the Hesse potential. However, in that case the result would no longer be consistent with the initial assumption that was made in section 5 , namely that we assumed from the beginning that $\Omega$ depends only on $S$ and $\bar{S}$, thus excluding any other additional terms in the effective action. This initial assumption was partly a matter of convenience, and it is difficult to fully exclude other starting points at this stage.

In closing we conclude that, generically, the Hesse potential may contain terms that are non-holomorphic in the topological string coupling constant, and that these do not exclusively originate from the non-holomorphic corrections in the effective action. However, due to lack of data on both the effective action and on the topological string side, we cannot at present draw a definite conclusion about the presence of such terms in the Hesse potential. Our findings do not, at this stage, contradict the idea that the Hesse potential could actually coincide with the topological string. Should this be the case, this will have calculable implications for the effective action, which in principle can be worked out explicitly by means of the iterative method proposed in this paper. 


\section{Acknowledgments}

We acknowledge helpful discussions with Albrecht Klemm, Thomas Grimm, Thomas Mohaupt, Ashoke Sen and Marcel Vonk. The work of G.L.C. is supported by the Fundação para a Ciência e a Tecnologia (FCT/Portugal) and by a partnership grant PHYS0167 of the Alexander von Humboldt Stiftung.

Open Access. This article is distributed under the terms of the Creative Commons Attribution Noncommercial License which permits any noncommercial use, distribution, and reproduction in any medium, provided the original author(s) and source are credited.

\section{References}

[1] G.L. Cardoso, B. de Wit, J. Kappeli and T. Mohaupt, Black hole partition functions and duality, JHEP 03 (2006) 074 [hep-th/0601108] [SPIRES].

[2] G.L. Cardoso, B. de Wit and S. Mahapatra, Subleading and non-holomorphic corrections to $N=2$ BPS black hole entropy, JHEP 02 (2009) 006 [arXiv:0808.2627] [SPIRES].

[3] M. Bershadsky, S. Cecotti, H. Ooguri and C. Vafa, Kodaira-Spencer theory of gravity and exact results for quantum string amplitudes, Commun. Math. Phys. 165 (1994) 311 [hep-th/9309140] [SPIRES].

[4] I. Antoniadis, E. Gava, K.S. Narain and T.R. Taylor, Topological amplitudes in string theory, Nucl. Phys. B 413 (1994) 162 [hep-th/9307158] [SPIRES].

[5] S. Ferrara, J.A. Harvey, A. Strominger and C. Vafa, Second quantized mirror symmetry, Phys. Lett. B 361 (1995) 59 [hep-th/9505162] [SPIRES].

[6] A. Sen and C. Vafa, Dual pairs of type-II string compactification, Nucl. Phys. B 455 (1995) 165 [hep-th/9508064] [SPIRES].

[7] A. Gregori, C. Kounnas and P.M. Petropoulos, Non-perturbative triality in heterotic and type-II $N=2$ strings, Nucl. Phys. B 553 (1999) 108 [hep-th/9901117] [SPIRES].

[8] T.W. Grimm, A. Klemm, M. Mariño and M. Weiss, Direct integration of the topological string, JHEP 08 (2007) 058 [hep-th/0702187] [SPIRES].

[9] H. Ooguri, A. Strominger and C. Vafa, Black hole attractors and the topological string, Phys. Rev. D 70 (2004) 106007 [hep-th/0405146] [SPIRES].

[10] G.L. Cardoso, B. de Wit and T. Mohaupt, Corrections to macroscopic supersymmetric black-hole entropy, Phys. Lett. B 451 (1999) 309 [hep-th/9812082] [SPIRES].

[11] L.J. Dixon, V. Kaplunovsky and J. Louis, Moduli dependence of string loop corrections to gauge coupling constants, Nucl. Phys. B 355 (1991) 649 [SPIRES].

[12] G.L. Cardoso, B. de Wit and T. Mohaupt, Macroscopic entropy formulae and non-holomorphic corrections for supersymmetric black holes, Nucl. Phys. B 567 (2000) 87 [hep-th/9906094] [SPIRES].

[13] J.A. Harvey and G.W. Moore, Fivebrane instantons and $R^{2}$ couplings in $N=4$ string theory, Phys. Rev. D 57 (1998) 2323 [hep-th/9610237] [SPIRES]. 
[14] G.L. Cardoso, B. de Wit, J. Kappeli and T. Mohaupt, Asymptotic degeneracy of dyonic $N=4$ string states and black hole entropy, JHEP 12 (2004) 075 [hep-th/0412287] [SPIRES].

[15] D.P. Jatkar and A. Sen, Dyon spectrum in CHL models, JHEP 04 (2006) 018 [hep-th/0510147] [SPIRES].

[16] R. Dijkgraaf, E.P. Verlinde and H.L. Verlinde, Counting Dyons in $N=4$ string theory, Nucl. Phys. B 484 (1997) 543 [hep-th/9607026] [SPIRES].

[17] D. Shih, A. Strominger and X. Yin, Recounting dyons in $N=4$ string theory, JHEP 10 (2006) 087 [hep-th/0505094] [SPIRES].

[18] J.R. David and A. Sen, CHL dyons and statistical entropy function from D1 - D5 system, JHEP 11 (2006) 072 [hep-th/0605210] [SPIRES].

[19] S. Banerjee, A. Sen and Y.K. Srivastava, Generalities of quarter BPS Dyon partition function and Dyons of torsion two, JHEP 05 (2008) 101 [arXiv:0802.0544] [SPIRES].

[20] S. Banerjee, A. Sen and Y.K. Srivastava, Partition functions of torsion $>1$ Dyons in heterotic string theory on $T^{6}$, JHEP 05 (2008) 098 [arXiv:0802.1556] [SPIRES].

[21] A. Dabholkar, J. Gomes and S. Murthy, Counting all dyons in $N=4$ string theory, arXiv:0803.2692 [SPIRES].

[22] G.L. Cardoso, B. de Wit, J. Kappeli and T. Mohaupt, Stationary BPS solutions in $N=2$ supergravity with $R^{2}$ interactions, JHEP 12 (2000) 019 [hep-th/0009234] [SPIRES].

[23] R.M. Wald, Black hole entropy is the Noether charge, Phys. Rev. D 48 (1993) 3427 [gr-qc/9307038] [SPIRES].

[24] T. Jacobson, G. Kang and R.C. Myers, On black hole entropy, Phys. Rev. D 49 (1994) 6587 [gr-qc/9312023] [SPIRES].

[25] V. Iyer and R.M. Wald, Some properties of Noether charge and a proposal for dynamical black hole entropy, Phys. Rev. D 50 (1994) 846 [gr-qc/9403028] [SPIRES].

[26] D.S. Freed, Special Kähler manifolds, Commun. Math. Phys. 203 (1999) 31 [hep-th/9712042] [SPIRES].

[27] D.V. Alekseevsky, V. Cortes and C. Devchand, Special complex manifolds, J. Geom. Phys. 42 (2002) 85 [math/9910091] [SPIRES].

[28] D. Shih and X. Yin, Exact black hole degeneracies and the topological string, JHEP 04 (2006) 034 [hep-th/0508174] [SPIRES].

[29] G.L. Cardoso, J.R. David, B. de Wit and S. Mahapatra, The mixed black hole partition function for the STU model, JHEP 12 (2008) 086 [arXiv:0810.1233] [SPIRES].

[30] S. Banerjee and R.K. Gupta, Duality covariant variables for STU-model in presence of non-holomorphic corrections, arXiv:0905.2700 [SPIRES].

[31] S. Chaudhuri, G. Hockney and J.D. Lykken, Maximally supersymmetric string theories in $D<10$, Phys. Rev. Lett. 75 (1995) 2264 [hep-th/9505054] [SPIRES].

[32] A. Gregori et al., $R^{2}$ corrections and non-perturbative dualities of $N=4$ string ground states, Nucl. Phys. B 510 (1998) 423 [hep-th/9708062] [SPIRES].

[33] J.A. Harvey and G.W. Moore, Exact gravitational threshold correction in the FHSV model, Phys. Rev. D 57 (1998) 2329 [hep-th/9611176] [SPIRES]. 
[34] A. Klemm and M. Mariño, Counting BPS states on the Enriques Calabi-Yau, Commun. Math. Phys. 280 (2008) 27 [hep-th/0512227] [SPIRES].

[35] R.E. Borcherds, Automorphic forms with singularities on Grassmannians, Invent. Math. 132 (1998) 491 [alg-geom/9609022]. 